University of Tennessee Health Science Center

UTHSC Digital Commons

\title{
An Innovative Testing Protocol to Study Foot and Ankle Kinetics during Early Stance Phase of Gait
}

Thomas E. Stewart

University of Tennessee Health Science Center

Follow this and additional works at: https://dc.uthsc.edu/dissertations

Part of the Investigative Techniques Commons

\section{Recommended Citation}

Stewart, Thomas E. , "An Innovative Testing Protocol to Study Foot and Ankle Kinetics during Early Stance Phase of Gait" (2012). Theses and Dissertations (ETD). Paper 252. http://dx.doi.org/10.21007/ etd.cghs.2012.0299.

This Thesis is brought to you for free and open access by the College of Graduate Health Sciences at UTHSC Digital Commons. It has been accepted for inclusion in Theses and Dissertations (ETD) by an authorized administrator of UTHSC Digital Commons. For more information, please contact jwelch30@uthsc.edu. 


\title{
An Innovative Testing Protocol to Study Foot and Ankle Kinetics during Early Stance Phase of Gait
}

\begin{abstract}
The objective of this study was to improve upon existing testing platform limitations with respect to foot and ankle mechanics in the sagittal plane during dorsiflexion and plantar flexion. The intent was to develop a multi-loading protocol that simulated aspects of early stance phase of walking gait. This data were used to evaluate the influence an Achilles load has on the kinematic profile of the ankle complex. Also, resulting kinematic profile data can be used to evaluate ligament/tendon effects, ankle arthroplasty, and various surgical techniques.

A pair of cadaveric human feet, from the same donor, 50 years of age were dissected and potted for testing. A pure moment protocol was developed to determine the path of least resistance or lowest energy state to rotate the tibia about the ankle complex. This protocol utilized a 4-degree of freedom robot coupled with a two 6-axis load cells. Positional data was used to calculate the instantaneous axis of rotation (IAR) of the ankle complex. The data was then normalized with respect to the widest distance across the tibia.

Results from this work include a repeatability study of the robotic testing platform (RTP), validation of protocol, calculation of the IAR, and a study of the effect an Achilles load has on ankle kinematics. The repeatability study used a modified version of the protocol to reduce setup effects. A repeatability analysis was conducted comparing repeated test runs for dorsiflexion and plantar flexion (one way repeated measures ANOVA with a Bonferroni test) and found no significant difference between the data sets for $(P<0.05)$. The IAR results with and without a passive Achilles load were significantly different $(P>0.05)$, using same statistical approach.
\end{abstract}

Future work is to actively drive the Achilles load and add a push-off condition were the rotation is about the distal end of the first and second metatarsals. Along with that, the upper limit of the vGRF is to be increased to simulate the later part of the stance phase of gait where the Achilles load is larger.

\section{Document Type}

Thesis

Degree Name

Master of Science (MS)

Program

Biomedical Engineering and Imaging

Research Advisor

Denis J. DiAngelo, Ph.D.

\section{Keywords}

ankle complex, four bar linkage, instantaneous axis of rotation, testing platform

\section{Subject Categories}

Analytical, Diagnostic and Therapeutic Techniques and Equipment | Investigative Techniques | Medicine and Health Sciences 


\title{
An Innovative Testing Protocol to Study Foot and Ankle Kinetics during Early Stance Phase of Gait
}

\author{
A Thesis \\ Presented for \\ The Graduate Studies Council \\ The University of Tennessee \\ Health Science Center
}

\author{
In Partial Fulfillment \\ Of the Requirements for the Degree \\ Master of Science \\ In the Joint Graduate Program in Biomedical Engineering and Imaging \\ From The University of Tennessee \\ and \\ The University of Memphis
}

By

Thomas E. Stewart

December 2012 
Copyright (C 2012 by Thomas E. Stewart. All rights reserved. 


\section{DEDICATION}

This thesis is dedicated to my loving and supportive wife (thank you Jennifer!) and my patient children, Samantha, Joshua, Robert, and Morgan. It was their love and support that kept me going. 


\section{ACKNOWLEDGEMENTS}

Thanks to my research advisor Dr. Denis DiAngelo for his tenacious drive and support of this work. His guidance throughout this experience has been vital to me finishing. Dr. Brian Kelly also deserves special acknowledgement for his repeated assistance in times of need. Also, I want to give a big thank you to Dr. Lewis and Dr. Kasser for their invaluable input and direction keeping my research on track and focused.

I have to thank the members of this lab for their support, even in times of tension. This team has undergone the typical dynamics of a team and pulled together when needed; thank you Kelly, Dan, Hunter, and John for your help these past few years. Also, Braham, Karen, and Jessica, thank you very much for your help.

A special thank you to my wife Jennifer for her faith in me even when I did not have faith in myself. Finally, I give thanks to my children for their endless reassuring with comments like "you can do this" and my favorite "you got this." 


\begin{abstract}
The objective of this study was to improve upon existing testing platform limitations with respect to foot and ankle mechanics in the sagittal plane during dorsiflexion and plantar flexion. The intent was to develop a multi-loading protocol that simulated aspects of early stance phase of walking gait. This data were used to evaluate the influence an Achilles load has on the kinematic profile of the ankle complex. Also, resulting kinematic profile data can be used to evaluate ligament/tendon effects, ankle arthroplasty, and various surgical techniques.
\end{abstract}

A pair of cadaveric human feet, from the same donor, 50 years of age were dissected and potted for testing. A pure moment protocol was developed to determine the path of least resistance or lowest energy state to rotate the tibia about the ankle complex. This protocol utilized a 4-degree of freedom robot coupled with a two 6-axis load cells. Positional data was used to calculate the instantaneous axis of rotation (IAR) of the ankle complex. The data was then normalized with respect to the widest distance across the tibia.

Results from this work include a repeatability study of the robotic testing platform (RTP), validation of protocol, calculation of the IAR, and a study of the effect an Achilles load has on ankle kinematics. The repeatability study used a modified version of the protocol to reduce setup effects. A repeatability analysis was conducted comparing repeated test runs for dorsiflexion and plantar flexion (one way repeated measures ANOVA with a Bonferroni test) and found no significant difference between the data sets for $(\mathrm{P}<0.05)$. The IAR results with and without a passive Achilles load were significantly different $(\mathrm{P}>0.05)$, using same statistical approach.

Future work is to actively drive the Achilles load and add a push-off condition were the rotation is about the distal end of the first and second metatarsals. Along with that, the upper limit of the vGRF is to be increased to simulate the later part of the stance phase of gait where the Achilles load is larger. 


\section{TABLE OF CONTENTS}

CHAPTER 1. INTRODUCTION ................................................................................

CHAPTER 2. BACKGROUND OF THE FOOT AND ANKLE ..................................4

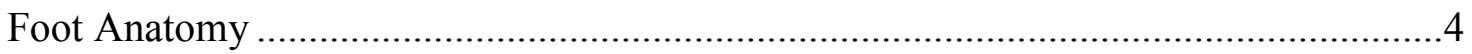

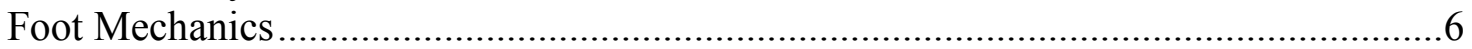

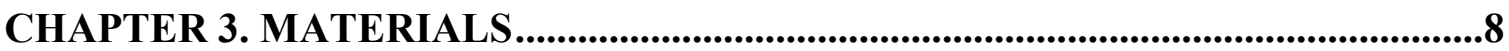

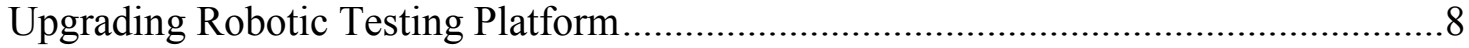

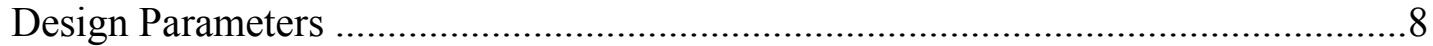

Establishing 3D CAD Model ..............................................................................

Modifications to Current Testing Platform ............................................................ 10

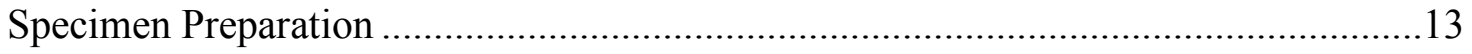

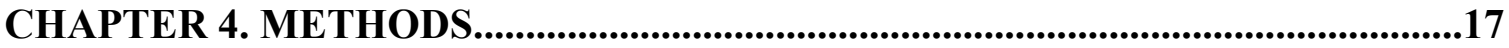

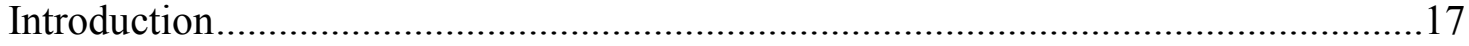

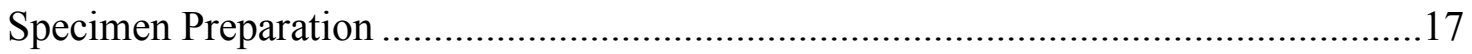

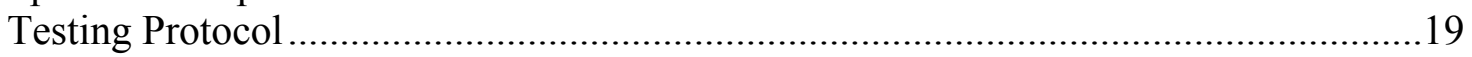

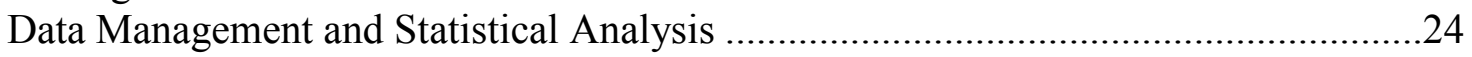

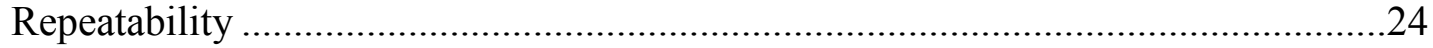

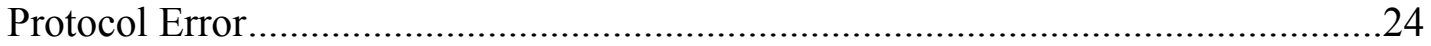

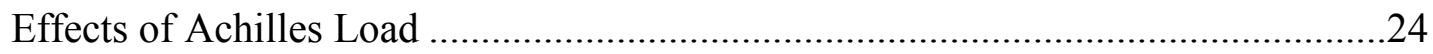

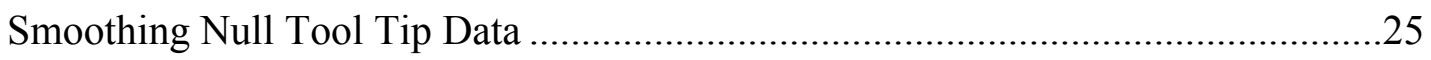

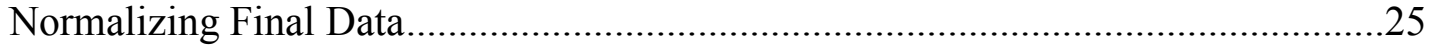

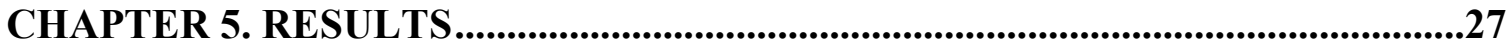

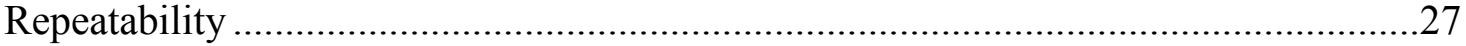

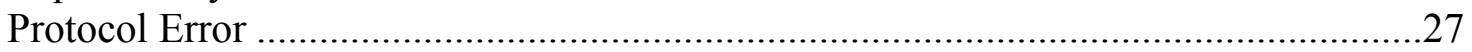

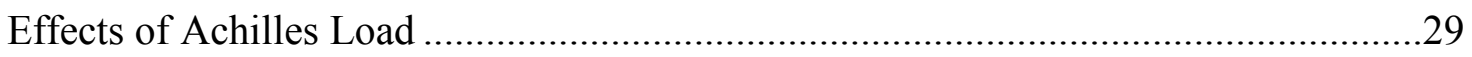

CHAPTER 6. AN INNOVATIVE TESTING PROTOCOL FOR THE FOOT AND ANKLE COMPLEX ..........................................................................................33

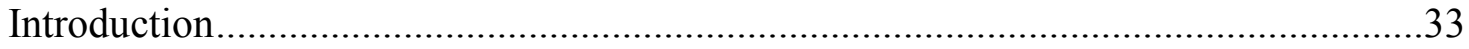

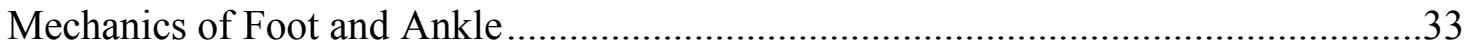

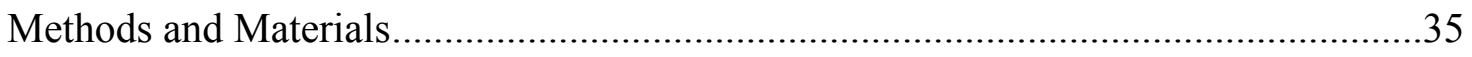

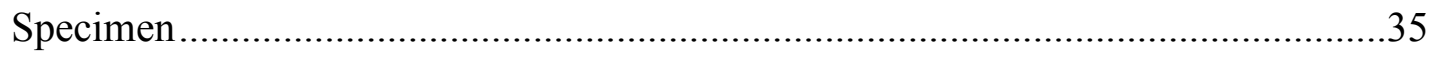

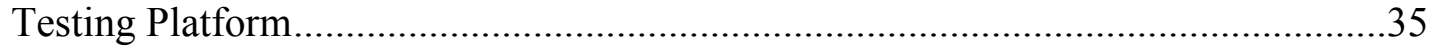

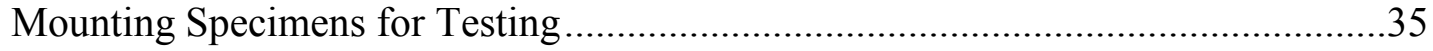

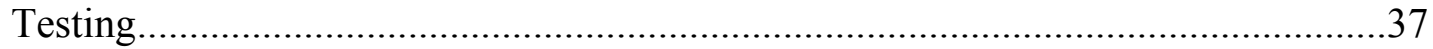

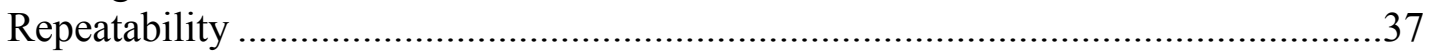

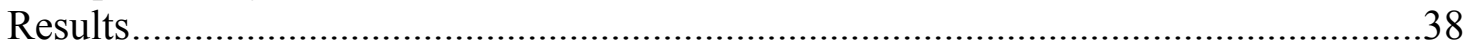

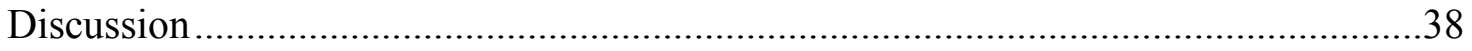

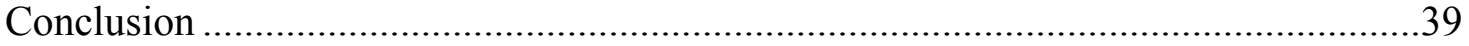


CHAPTER 7. DISCUSSION ......................................................................................41

CHAPTER 8. CONCLUSION ..............................................................................................43

CHAPTER 9. LIMITATIONS AND FUTURE WORK ..............................................44

LIST OF REFERENCES.................................................................................................45

APPENDIX A. GENERIC GAIT DATA.............................................................................47

APPENDIX B. TRANSFORMATIONS, TOOL TIP AND FORCE FRAME............50

APPENDIX C. DATA PROCESSING, POLYNOMIAL FIT, SMOOTHING, AND IAR CALCULATIONS .....................................................................................51

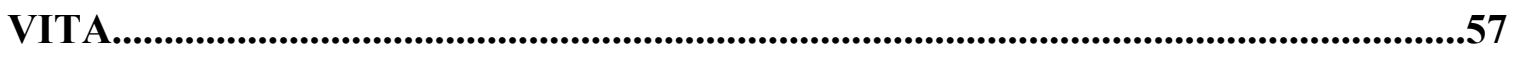




\section{LIST OF TABLES}

Table 2-1. Key components of stance phase and loading characteristics. .....................7

Table 3-1. Previous RTP degrees of freedom with ROM and loading limits...............10

Table 3-2. Comparison between rotary actuators, new versus existing. ......................11

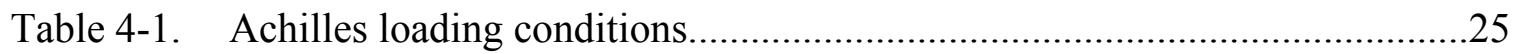

Table 5-1. Protocol error induced based on angular step size................................28 


\section{LIST OF FIGURES}

Figure 1-1. Platform from Iowa State University and University of Salford..................2

Figure 1-2. Platform from Pennsylvania State University........................................2

Figure 1-3. Platform from Cleveland Clinic..........................................................

Figure 1-4. Platform from University of Wisconsin-Milwaukee and Mayo Clinic. ..........3

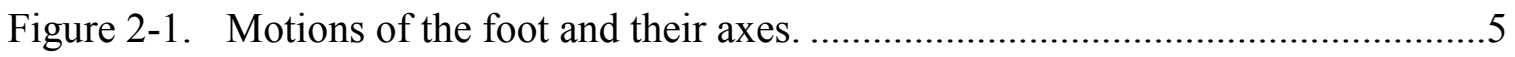

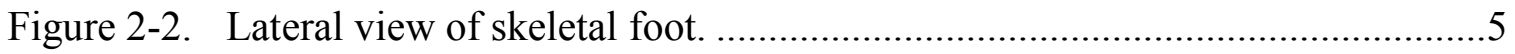

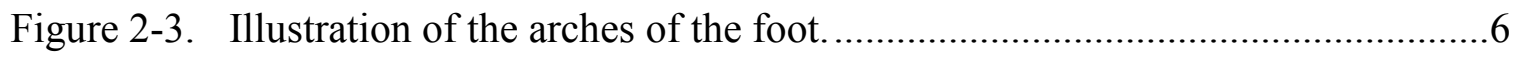

Figure $2-4 . \quad$ Free body diagram of foot and ankle. ….............................................

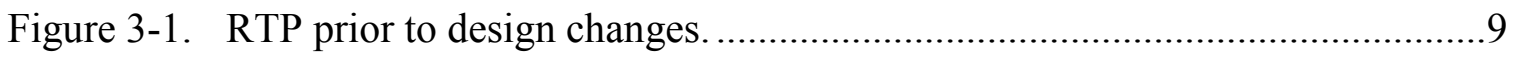

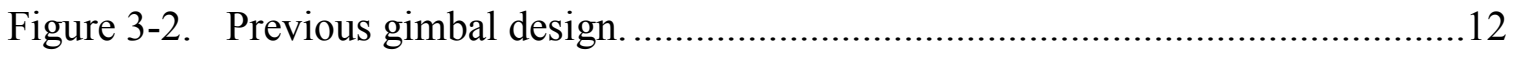

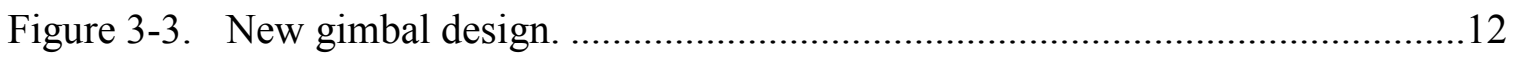

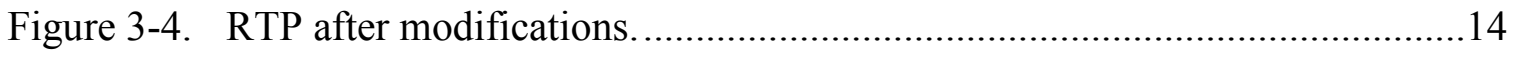

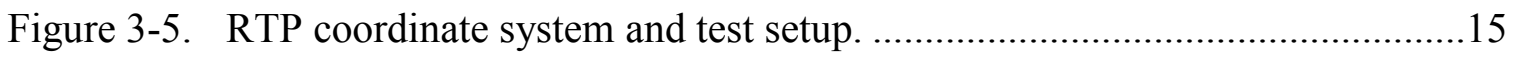

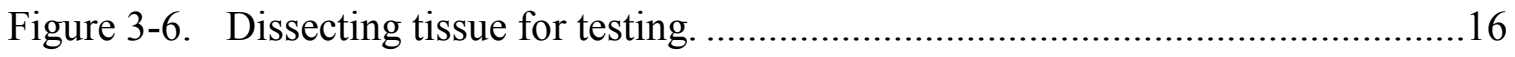

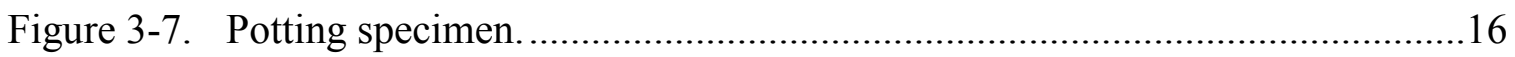

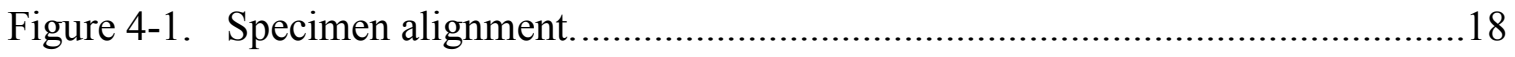

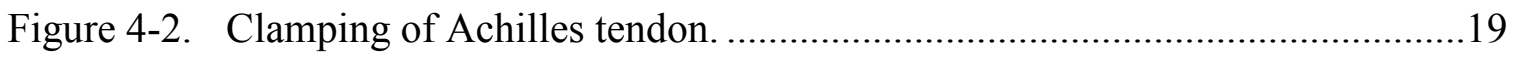

Figure 4-3. Incremental step from test protocol. .................................................20

Figure 4-4. Initial center of rotation, needed for protocol. ....................................22

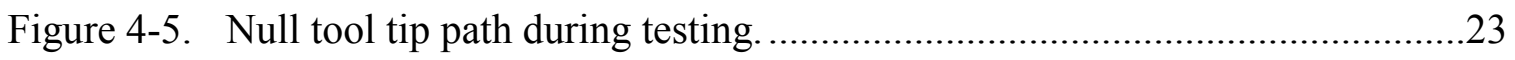

Figure 4-6. IAR established by intersecting perpendicular bisectors........................23

Figure 4-7. Null tool tip data before smoothing process. ........................................26

Figure 4-8. Normalized chart for plotting various IAR results. ...............................26

Figure 5-1. IAR curves used to evaluate repeatability of RTP and protocol.................27 


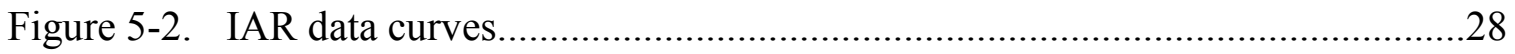

Figure 5-3. IAR data for specimens 1 and 2 at various loads on Achilles .......................30

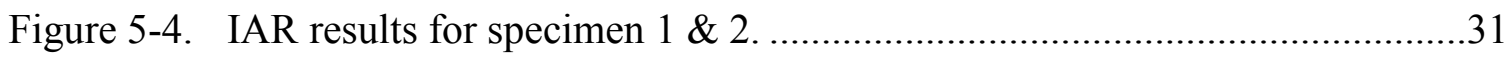

Figure 5-5. Image from Leardini et al showing IAR path..............................................31

Figure 5-6. Image from Baxter et al showing single point approximation.......................32

Figure 6-1. Free body diagram of foot and ankle complex. .............................................34

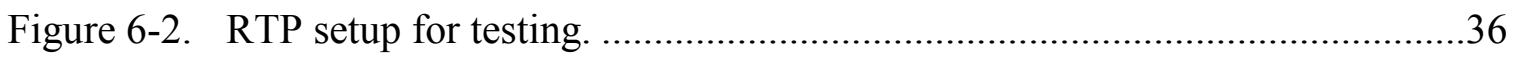

Figure 6-3. Null tool tip and extended tool tip..............................................................

Figure 6-4. Illustration of incremental step in custom testing program. .........................38

Figure 6-5. Illustration of IAR pathway. ....................................................................

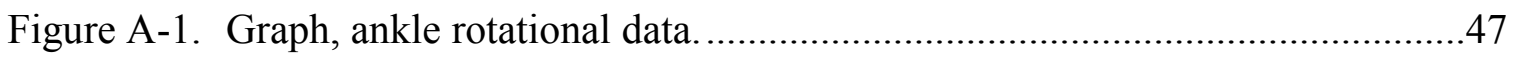

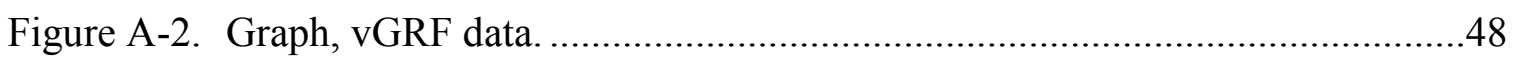

Figure A-3. Graph, moment at ankle.....................................................................49

Figure B-1. Tool tip transformations, dimensions in $\mathrm{mm}$...........................................50 


\section{CHAPTER 1. INTRODUCTION}

In 2010, it was estimated that two million Americans sought medical attention for ankle pain [1]. Major sources for this pain were arthritis, post-traumatic injury, and loss of medial arch resulting from trauma or complications from diabetes. Of those patients, 50,000 were expected to be diagnosed with end stage arthritis and 4,000 total ankle replacements (TARs) were performed. With the "Baby Boomers" aging these numbers are only expected to rise in the coming years.

Surgical treatment of the ankle joint is problematic due to its inherent complexity. For severe ankle pain, resulting from disease or trauma, common medical procedures are TAR or ankle arthrodesis. Since TARs had high failure rates when introduced in 1970, arthrodesis became the gold standard for major ankle aliments causing server pain. Short comings of ankle arthrodesis are its impact on gait mechanics and the transference of non-physiological forces to adjacent joints. Although TARs are much better today they are still less trusted as a treatment when compared to arthrodesis. Gougoulias et al [2] reported major failures for TARs as misalignment of implant, loosening of components (largely tibial components), and improper mechanics (implant did not behave like an ankle joint). It is critical that the kinematics of the ankle complex be accurately studied so both treatments can be evaluated and so the effects of each are known.

Flexion and extension of the ankle joint has a unique kinematic pathway that it follows. This path is often referred to as the instantaneous axis of rotation (IAR) pathway. In 1973 Sammarco et al [3] confirmed that the IAR of a normal ankle does not remain constant with motion and that it lies within the cross-sectional area of the talus as viewed in the sagittal plane. Leardini et al $[4,5,6,7]$ furthered this concept by modeling and simulating the ankle as a 4-bar linkage system and comparing it against in vitro test data. The results indicate that the human ankle behaves more like a 4-bar linkage model and less like a hinge joint. Clearly, predicting or measuring the IAR of an ankle would be help to understand effects of misalignment and improper mechanics.

Few testing platforms can evaluate lower limb kinetics $[8,9,10,11,12]$, and of those the instantaneous axis of rotation (IAR) is approximated or forced to follow a set IAR pathway that is not its own, refer to Figures 1-1 thru 1-4. Even with strong evidence that the ankle behaves according to a system with an instantaneous axis, many still approximate a single point for the ankle during rotation [13] or use overly simplified techniques as graphically tracking landmarks over large angles [14] on an x-ray. Hence, there is scope for development of a new, validated testing platform.

The objective going forward was to develop robotic testing platform (RTP) and testing protocol to evaluate foot and ankle mechanics with and without a passive Achilles force. The testing protocol was based on a pure moment test to establish the "path of least resistance" or lowest energy state to rotate the tibia about the ankle complex. Then study the kinetics of the ankle complex with and without an Achilles load and compare the results. 


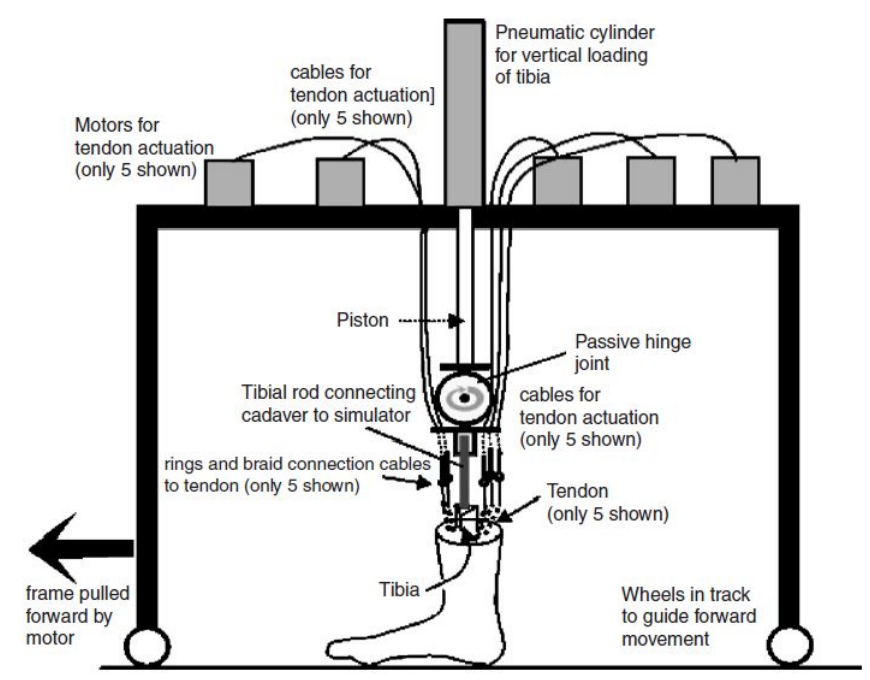

Figure 1-1. Platform from Iowa State University and University of Salford. Simulates active muscles to produce a gait cycle and has to take iterative steps to establish vGRF. Platform and has no way to determine IAR. Reprinted with permission. Nester, C.J., Liu, A.M., et al. (2007). In vitro study of foot kinematics using a dynamic walking cadaver model. J Biomech 40(2): 1927-1937.

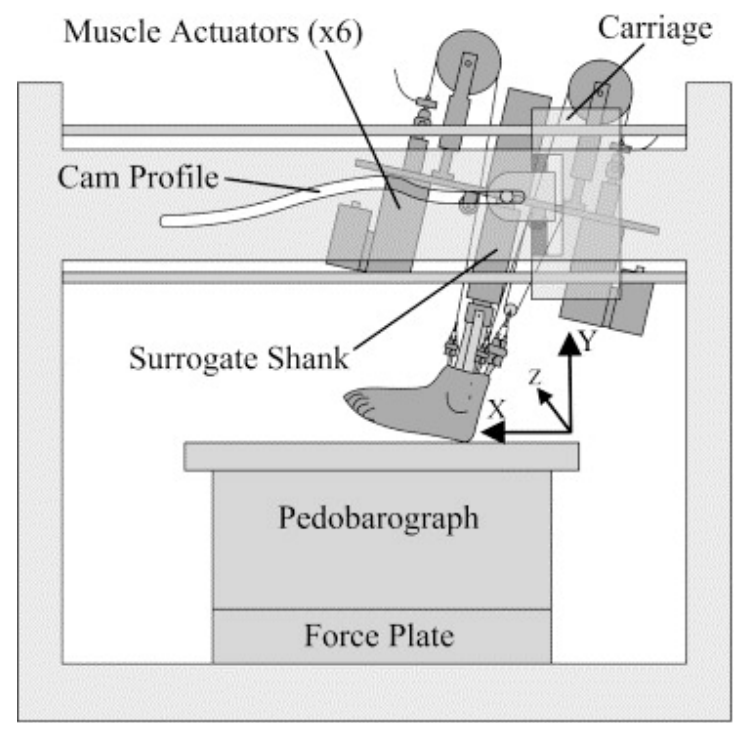

Figure 1-2. Platform from Pennsylvania State University.

Specimen follows a set cam profile and establishes vGRF by trial and error. IAR is forced to follow a kinematic profile that is not its own. Reprinted with permission. Hamil, A. J., et al. (2004). Relative motions of the tibia, talus, and calcaneus during the stance phase of gait: a cadaver study. Gait \& Posture. 20(2): 147-153. 


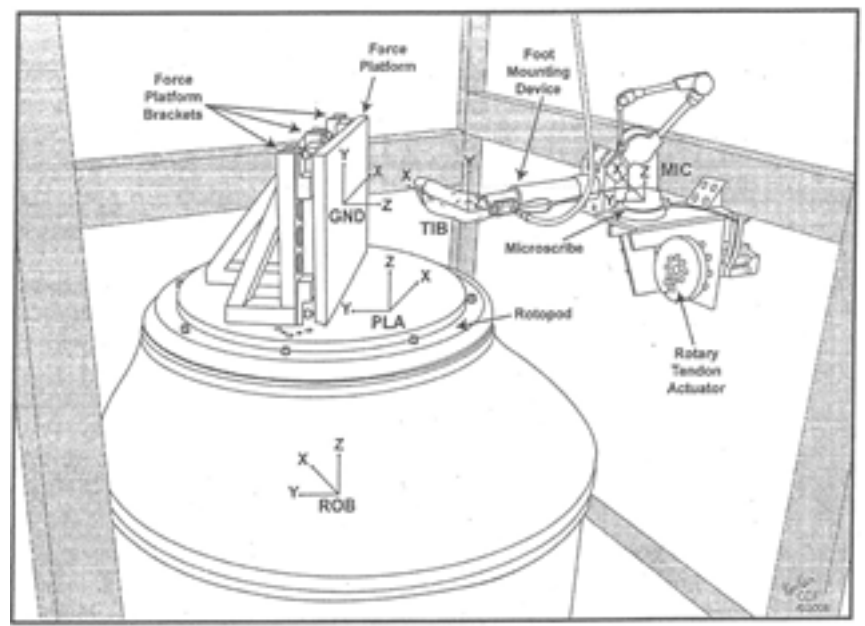

Figure 1-3. Platform from Cleveland Clinic.

This platform takes iterative steps to establish vGRF and specimen follows a predefined ground motion. Platform and has no way to determine IAR. Reprinted with permission. Noble, L., Colbrunn, R., et al. (2010). Design and Validation of a General Purpose Robotic Testing System for Musculoskeletal Applications, Journal of Biomechanical Engineering-Transactions of the ASME, 132(2).

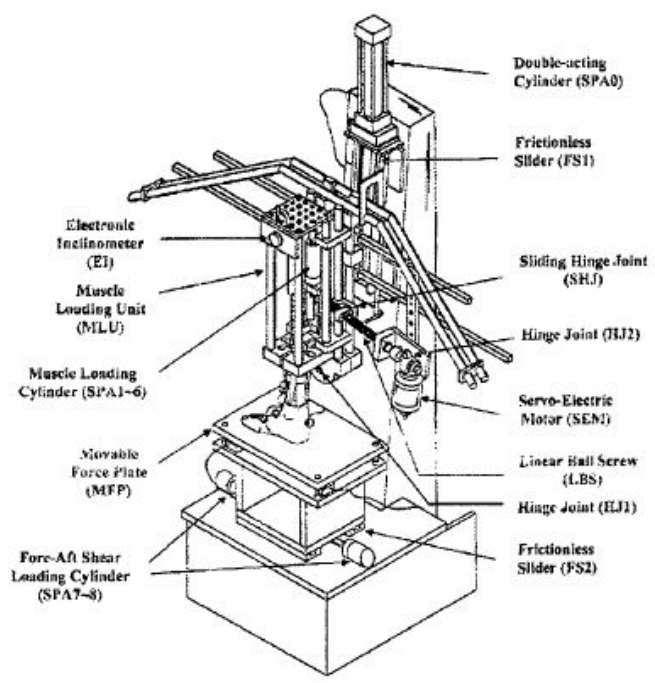

Figure 1-4. Platform from University of Wisconsin-Milwaukee and Mayo Clinic. Platform uses a four bar mechanism to provide sagittal plane rotation of tibia about the ankle joint. IAR is forced to follow a kinematic profile that is not its own. Reprinted with permission. Kim, K., Kitaoka,H.B., et al. (2001). In Vitro Simulation of the Stance Phase in Human Gait. Journal of Musculoskeletal Research, 5(6): 113-122. 


\section{CHAPTER 2. BACKGROUND OF THE FOOT AND ANKLE}

\section{Foot Anatomy}

The human foot is a complex biomechanical structure essential for normal locomotion. There are three motions in which the foot moves, each with its own axis and plane of motion, dorsiflexion-plantar flexion, abduction-adduction, and eversioninversion as shown in Figure 2-1. The ankle joint, made up of the tibia, talus, and fibula bones, provides the "hinge like" movement that allows the foot to propel the body forward during stance phase of gait. The ankle joint endures repetitive loading with dynamic loads reported at 4 to 5 times body weight [15] for certain activities.

The foot and ankle are made up of 26 bones, 33 joints and over 100 ligaments and tendons. The foot is clinically referred to as being comprised of three sections: forefoot, mid-foot, and hind-foot. The metatarsus and phalanges constitute the forefoot, cuboid, navicular, and cuneiforms the mid-foot, and talus and calcaneus the hind-foot (refer to Figure 2-2).

The bones of the hind-foot are components of the ankle joint (talocrural) and subtalar joint (talocalcaneal). The talus bone is common in both joints whereas the calcaneus is in the subtalar and not the ankle joint. Together they form the ankle complex. It is the ankle complex that is most relevant to this study.

The ankle joint is a synovial joint where the distal ends of the tibia and fibula meet with the proximal end of the talus. The tibia transfers the majority of the load (more than $80 \%$ ), as reported by Nordin and Frankkel [2], to the talus. The load transfer is made via articular surfaces, the superior surface of the talus and inferior surface of the tibia. This joint is responsible for dorsiflexion and plantar flexion. The major muscles that drive these motions are the tibialis anterior and gastrocnemius/soleus respectively. Typical range of motion (ROM) for this joint is 10 to $20^{\circ}$ dorsiflexion and 40 to $55^{\circ}$ plantar flexion [2].

The subtalar joint is also a synovial joint formed by the articulation of the calcaneus and talus, which articulate anteriorly and posteriorly. Clinically, the talocalcaneonavicular joint is considered part of the subtalar joint because these joints articulate together to induce an inversion or eversion motion.

The subtalar joint is activated by muscles inserting on the Achilles tendon. These muscles are located in the posterior compartment in the leg. The plantaris, gastrocnemius, and soleus insert into the posterior aspect of the calcaneus via the Achilles tendon. A model by Giddings et al [16] predicted loads through the Achilles tendon as high as 3.9 times BW during walking and 7.7 times BW while running.

There are three arches in the foot: the medial longitudinal arch, lateral longitudinal arch and transverse arch refer to Figure 2-3. The medial and lateral 


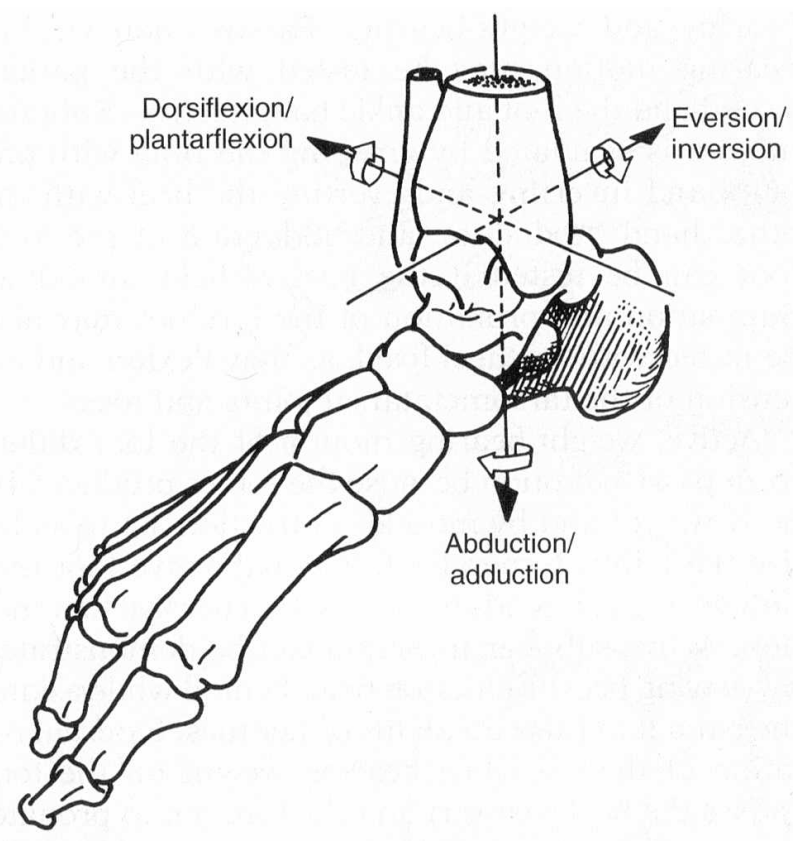

Figure 2-1. Motions of the foot and their axes.

Reprinted with permission. Nordin, M. and V.H. Frankel. (2001). Basic Biomechanics of the Musculoskeletal System. 3rd ed. Philadelphia, Lippincott Williams \& Wilkins. p. 226.

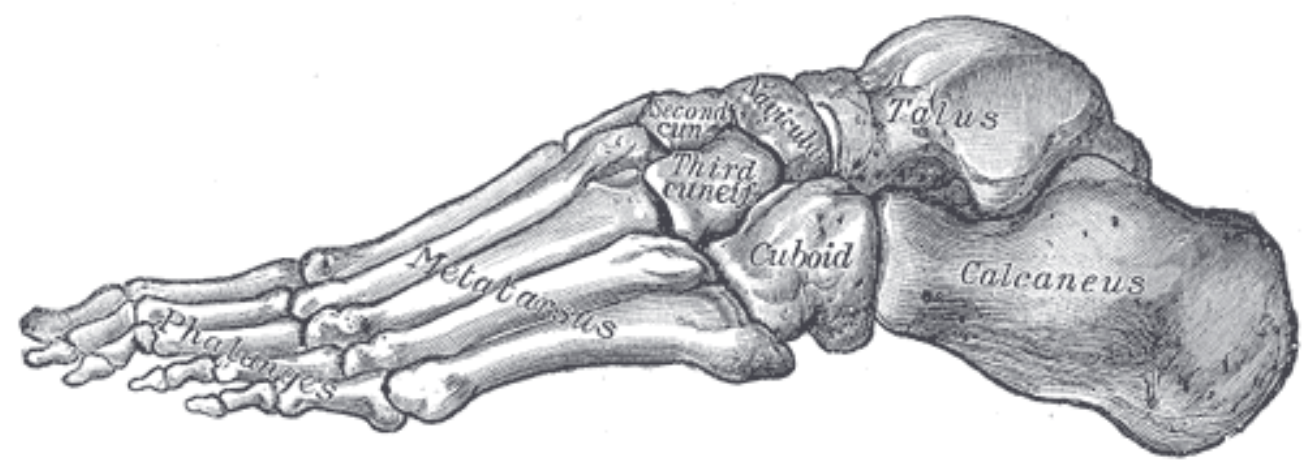

Figure 2-2. Lateral view of skeletal foot.

Reprinted with permission. Gray, H. and W. H. Lewis. (1918). Anatomy of the Human Body. Philadelphia, Lea \& Febiger. p.268. 


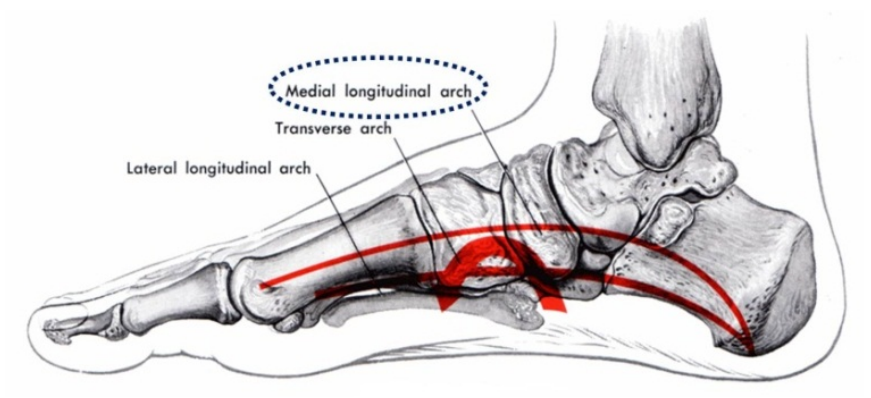

Figure 2-3. Illustration of the arches of the foot.

Reprinted with permission. Ortho-Worldwide. (2012). Ankle and Foot Bio-Mechanics. http://www.ortho-worldwide.com/anfobi.html. Accessed June 1, 2012.

longitudinal arches work together to transfer loads from the tibia down and to the calcaneus and metatarsals. It is this anterior and posterior load transfer that allows us to stand in an erect manner and maintain balance. However, balance would not be possible without the support and strength of the ligaments and tendons of the foot. In particular the plantar aponeurosis (part of the plantar fascia), which connects the calcaneus and metatarsals and thus supports the longitudinal arches of the foot with a "spring like" function. These arches are inherently strong and enable the foot to withstand high loads without damage.

\section{Foot Mechanics}

Human gait is any method of locomotion via loading and unloading of the involved limbs in a cyclic manner. For this study, gait events analyzed were those related to walking and the effects it has on the foot and ankle complex. Gait pattern for walking is viewed clinically as having two distinct phases, stance and swing [17]. Stance phase is the interval of gait in which the foot is in contact with the ground and is approximately $60 \%$ of the gait cycle [17]. The key components of stance phase (and loading characteristics) are listed in Table 2-1.

Gait data presented by Winter [18] describes the dynamic loading characteristics of the ankle. Winter's data were established via force plate measurements and tracking optical markers. Appendix A, incorporates this data into Excel ${ }^{\mathrm{TM}}$ and charts it accordingly. Changes in rotation, vGRF, and moment at the ankle during stance phase of gait are displayed with the key components from Table 2-1 identified. With this data a rigid body analysis can be used to establish an Achilles force $\left(\mathrm{F}_{\mathrm{a}}\right)$ and resulting force acting on the tibia. This model, shown in Figure 2-4, was used to establish testing parameters for both the RTP and protocol being developed for evaluating foot and ankle mechanics. 
Table 2-1. Key components of stance phase and loading characteristics.

\begin{tabular}{lcccc}
\hline Key Components & \% GC & $\begin{array}{c}\text { Ankle Rot. } \\
\left({ }^{\circ}\right)\end{array}$ & $\begin{array}{c}\text { vGRF } \\
(\mathrm{N})\end{array}$ & $\begin{array}{c}\mathbf{M}_{\mathbf{a}} \\
(\mathrm{Nm})\end{array}$ \\
\hline Heel Contact (HC) & 0 & -0.4 & -82.40 & -1.70 \\
Foot Flat (FF) & 16 & -0.6 & -550.20 & -5.10 \\
Heel Rise (HR) & 30 & 5.3 & -378.20 & -33.50 \\
Push Off (PO) & 46 & 5.9 & -602.80 & -89.70 \\
Toe Off (TO) & 60 & -20.1 & 3.50 & 1.40 \\
\hline
\end{tabular}

Data compiled from: Winter, D.A. (2009). Biomechanics and Motor Control of Human Movement. 4th ed. John Wiley \& Sons, Inc., Hoboken, NJ.

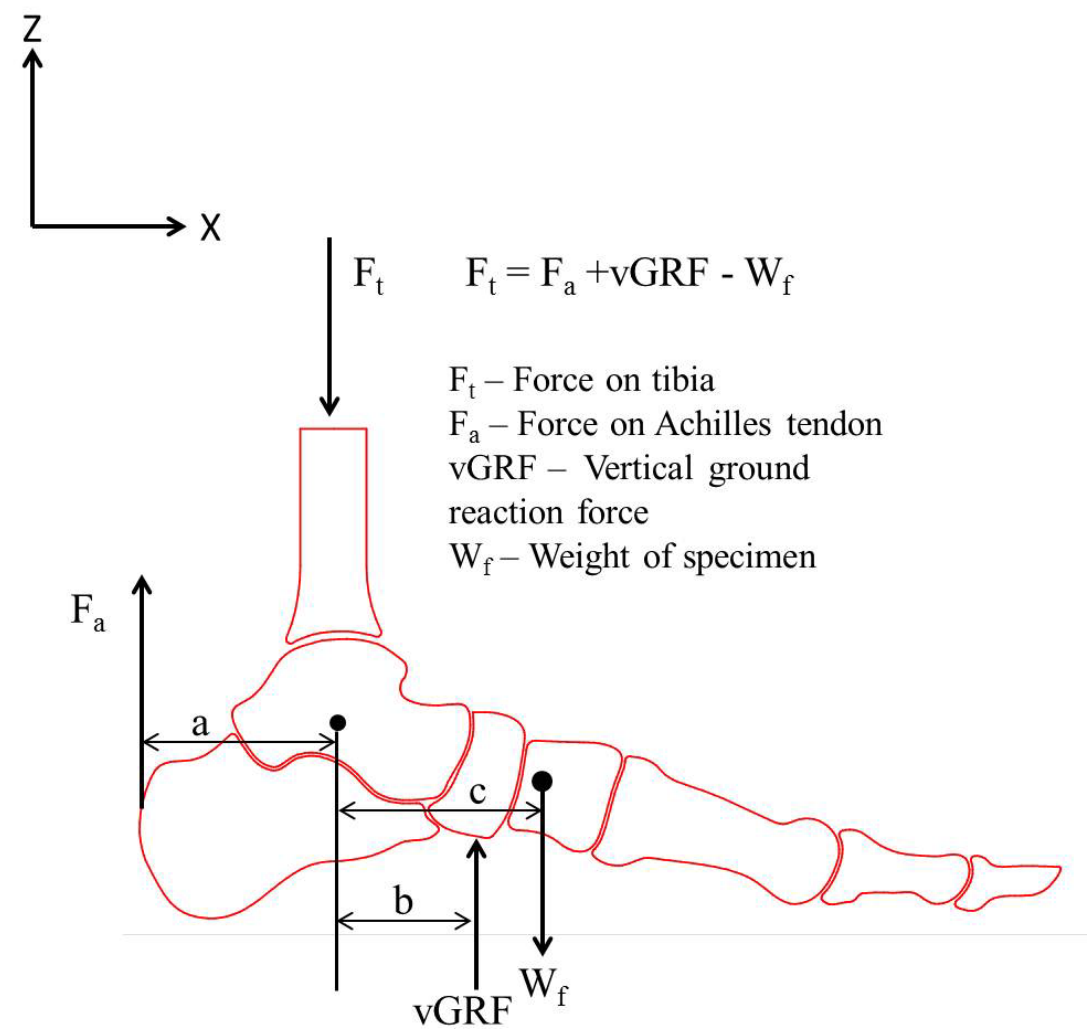

Figure 2-4. Free body diagram of foot and ankle.

The ankle is shown in a neutral position with the tibia normal to the ground. Also, the longitudinal arch is shown, including the bones that form the arch. 


\section{CHAPTER 3. MATERIALS}

\section{Upgrading Robotic Testing Platform}

The Robotic Testing Platform (RTP) used in the BioRobotics Laboratory at the University of Tennessee Health Science Center, see Figure 3-1, has been used primarily for testing and evaluating spine mechanics over the years. It has 4 degrees of freedom (DOF), reference Table 3-1. The RTP can drive joint motion in a plane, normally the sagittal plane of the joint being tested. It is programmable for load control and displacement control. It can monitor two 6-axis load cells and has the functionality to be upgraded to utilize 6 DOF plus command multiple robots.

The loading characteristics of the foot and ankle are more demanding and require an increased loading capacity during testing. The ankle experiences an approximate load of $600 \mathrm{~N}$ axially and a moment of $80 \mathrm{Nm}$ during normal walking [18]. The RTP was limited to $10 \mathrm{Nm}$ of continuous output from the rotary actuators that apply torque about the $\mathrm{Y}$ and $\mathrm{Z}$ axes. Existing linear actuators are capable of $2700 \mathrm{~N}$ minimum peak thrust force and/or carrying capacity [19] and are sufficient for foot and ankle testing.

\section{Design Parameters}

In order to replicate internal loads at the ankle as experienced during stance phase of gait the following design parameters were established:

- Max Achilles load (limited by tendon clamping technique)

- Minimum of $80 \mathrm{Nm}$ (result from Achilles load)

- $710 \mathrm{~N}$ tibia load (355 N vGRF and $355 \mathrm{~N}$ Achilles load)

- Up to $10^{\circ}$ of dorsiflexion

- Up to $20^{\circ}$ of plantar flexion

- Passively apply Achilles load

\section{Establishing 3D CAD Model}

The design parameters established for normal walking gait required redesign of several key components, largely the gimbal subassembly. To ensure proper fit and function the existing RTP was modeled in NX 6 (Siemens PLM software, Plano, TX). This gave a base model to design new parts form. The RTP was documented by way of 3D model and component drawings. 


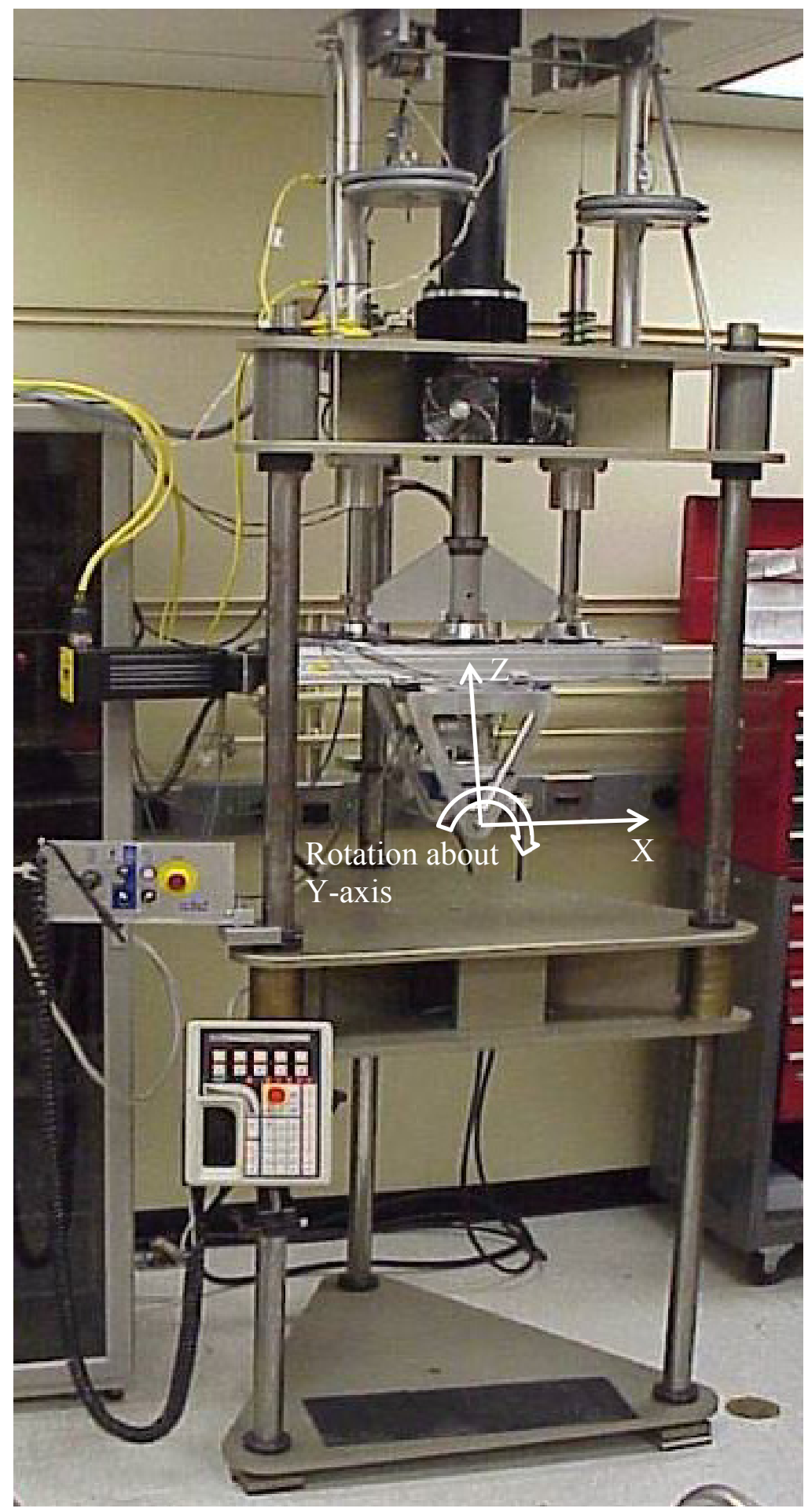

Figure 3-1. RTP prior to design changes.

Source: Modified with permission. Kelly, B. (2005). A Multiaxis programmable spine robot for the study of multibody spinal biomechanics using real-time hybrid force and displacement control strategies. Dissertation. The University of Tennessee Health Science Center, Memphis, TN. p. 60. 
Table 3-1. Previous RTP degrees of freedom with ROM and loading limits.

\begin{tabular}{cccc}
\hline Actuator Type & ROM & Applied Load & $\begin{array}{c}\text { Directional } \\
\text { Loading }\end{array}$ \\
\hline Rotary & $360^{\circ}$ & $10 \mathrm{Nm}$ & radial about Y axis \\
Rotary & $360^{\circ}$ & $10 \mathrm{Nm}$ & radial about $Z$ axis \\
Linear & $300 \mathrm{~mm}$ & $4450 \mathrm{~N}$ & linear along Z axis \\
Linear & $600 \mathrm{~mm}$ & $890 \mathrm{~N}$ & linear along X axis \\
\hline
\end{tabular}

\section{Modifications to Current Testing Platform}

To achieve the design parameters several changes had to be made. The current rotary actuator did not have the necessary power to drive the ankle through flexion during testing. Load requirements demanded an actuator with enough power to generate and hold an $80 \mathrm{Nm}$ load, an $800 \%$ increase to the RTP. An $80 \mathrm{Nm}$ load is a common load experienced at the ankle during gait. After researching several options, the FHA-25C160-US250 from Harmonic Drive, LLC (Peabody, MA) was chosen. Its specifications are compared against existing rotary actuators of the RTP in Table 3-2. In addition, Harmonic Drive actuators experience little to no backlash when rotating. This allows for smoother motion and better rotational data, especially when changing directions or accelerating.

The gimbal was redesigned to interface with the new FHA-25C-160 rotary actuators and meet the increased loading conditions stated above. Taking the same approach as before[19], maximum loading conditions were considered in designing the new gimbal. Maximum loading conditions were:

- $890 \mathrm{~N}$ along $\mathrm{X}$ axis

- $4450 \mathrm{~N}$ along $\mathrm{Z}$ axis

- $80 \mathrm{Nm}$ about $Z$ axis

- $80 \mathrm{Nm}$ about $\mathrm{Y}$ axis

Gimbal changes were significant as can be seen when comparing Figure 3-2 to Figure 3-3. The previous gimbal design rotated about the $\mathrm{Y}$ axis (also known as the pitch axis) as it was located below the load cell. This required an inter-frame and resulted in a unique rotational path. For the new gimbal design, a "yoke style" approach was taken which eliminated the inter-frame and allowed the pitch axis to rotate in line with the rotary actuator. This made it possible to establish a direct drive coupling with the actuator. With this design the outer frame increased in thickness. These changes added to the overall strength of the new gimbal to ensure new loading conditions during testing would not result in a part/assembly failure. A side effect of this change was the new location of the pitch axis. This required programing changes in tool tip transformations and moment corrections (from moment and shear errors that arise from fixture weight as test specimens are being rotated) in all RTP testing programs, reference Appendix B. 
Table 3-2. Comparison between rotary actuators, new versus existing.

\begin{tabular}{|c|c|c|c|c|c|c|c|c|}
\hline \multirow{2}{*}{$\begin{array}{c}\text { Rotary } \\
\text { Actuator }\end{array}$} & \multicolumn{2}{|c|}{ Torque } & \multirow[b]{2}{*}{$\begin{array}{c}\text { Resolution } \\
\left(\text { pulse } /{ }^{\circ}\right)\end{array}$} & \multirow[b]{2}{*}{$\begin{array}{c}\text { ROM } \\
\left(^{\circ}\right)\end{array}$} & \multirow[b]{2}{*}{$\begin{array}{c}\text { Current } \\
\text { (amps) }\end{array}$} & \multicolumn{3}{|c|}{ Allowable Load } \\
\hline & $\begin{array}{l}\text { Peak } \\
(\mathrm{Nm})\end{array}$ & $\begin{array}{l}\text { Continuous } \\
(\mathrm{Nm})\end{array}$ & & & & $\begin{array}{l}\text { Axial } \\
(\mathrm{N})\end{array}$ & $\begin{array}{l}\text { Radial } \\
(\mathrm{N})\end{array}$ & $\begin{array}{c}\text { Moment } \\
(\mathrm{Nm})\end{array}$ \\
\hline $\begin{array}{c}\text { Kollmorgen } \\
\text { 9FG }\end{array}$ & 16 & 10 & 0.0045 & 360 & 5.2 & 22 & 111 & NA \\
\hline $\begin{array}{c}\text { Harmonic Drive } \\
\text { FHA-25C-160 }\end{array}$ & 260 & 80 & 0.000225 & 360 & 4 & 14,700 & 4,900 & 370 \\
\hline
\end{tabular}

Data compiled form Harmonic Drive LLC. (2011). Hollow Shaft Actuators, FHA C series User Manual Catalog. Rev 08. Peabody, MA. and Kollmorgen Motions Technologies Group. (2006). Servo Disc Catalog. Commack, NY. 


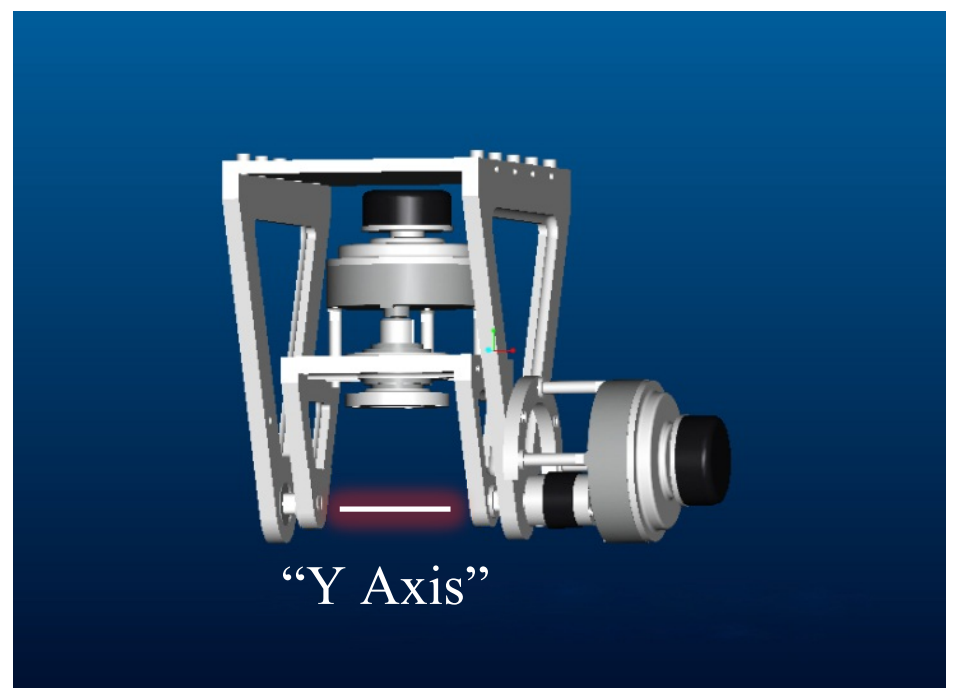

Figure 3-2. Previous gimbal design.

Source: Modified with permission. Kelly, B., A Multiaxis programmable spine robot for the study of multibody spinal biomechanics using real-time hybrid force and displacement control strategies. Dissertation, 2005. The University of Tennessee Health Science Center, Memphis. p. 55.

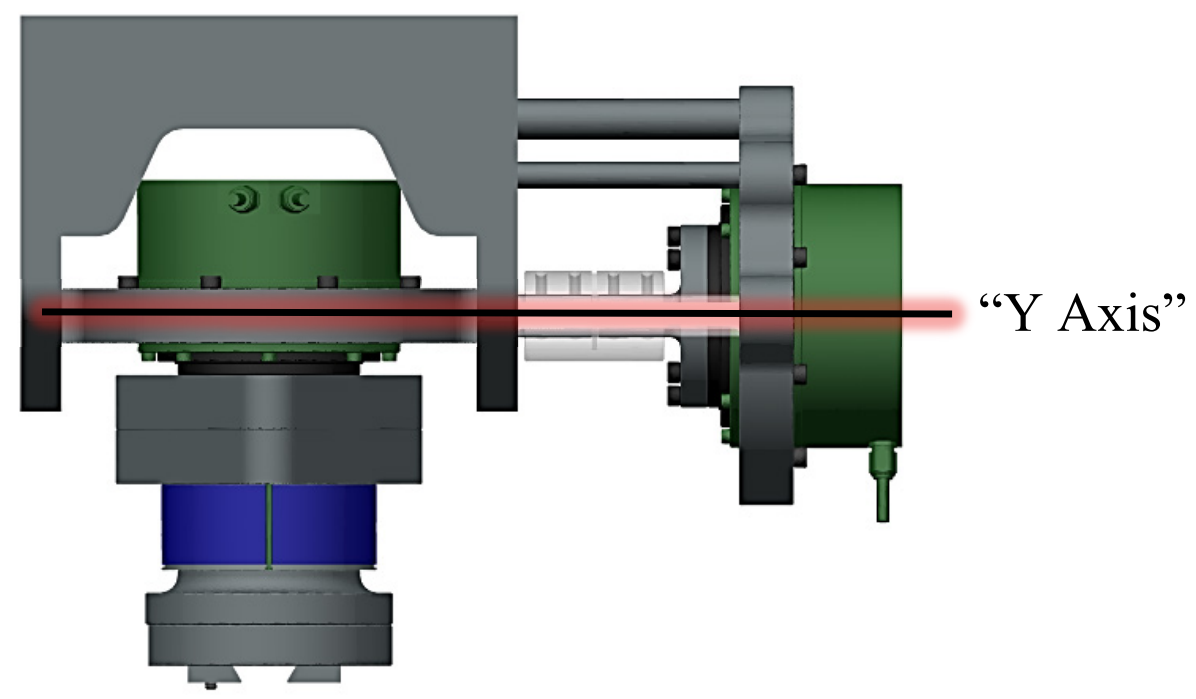

Figure 3-3. New gimbal design.

This figure illustrates the alignment of $\mathrm{Y}$ axis with center axis of the rotary actuator that controls the pitch motion for the RTP. This is a direct-drive connection drive for rotation about the $\mathrm{Y}$ axis of RTP. 
The horizontal linear actuator was rotated 90 degrees about the Z-axis to improve overall functionality when testing various specimen types. This also changed accessibility when mounting specimens and made it possible to position a c-arm around a test specimen during testing. Refer to Figure 3-4 to see the RTP after changes and Figure 3-5 to view an illustration of protocol test setup.

New components were assembled to the RTP with no fit-up issues. New digital servo drives and rotary actuators were tuned and specification files created to allow existing Adept controller (encoder and servo) to command these new devices. Validation tests were conducted to ensure form, fit and function of changes. The Adept controller commanded the new actuators without an issue and the new gimbal duplicated the range of motion of the old gimbal in all aspects except additional linear travel along the $\mathrm{X}$-axis was needed as the point of rotation increased in distance from the pitch axis.

The RTP has a positional accuracy of $2 \mu \mathrm{m}$ in the X-axis and $0.31 \mu \mathrm{m}$ in the Zaxis [19]. The rotary actuator used to rotate about ( $\mathrm{Y}$ and $\mathrm{Z}$ axes) has a resolution of $0.0002^{\circ}$, refer to Figure 3-4. The load cells used with the RTP measure the following forces: $F_{x}, F_{y}, F_{z}, M_{x}, M_{y}$, and $M_{z}$. They can be used together or one at a time based on the needs of testing. The resolution for the gimbal and base load cells are $0.4 \mathrm{~N}$ and $1.5 \mathrm{~N}$ respectively.

The positional accuracy of the null tool tip (NTT) is within $2.02 \mu \mathrm{m}$ and is recorded verses time and step count. Since the extended tool tip (ETT) is treated as it is rigidly connected to the NTT, its exact position was recorded in the same format and with the same accuracy, refer to Figure 3-4.

\section{Specimen Preparation}

A matched pair of feet (male, age 50) was used for this study. The specimens were stored at $-20^{\circ} \mathrm{C}$ until being prepped for testing. Each was cut to approximately 254 $\mathrm{mm}$ in height. Muscle and soft tissue was dissected and removed to expose $76.2 \mathrm{~mm}$ of the tibia and fibula at the top of each specimen, see Figure 3-6. Clearance and engagement holes were predrilled into the tibia and fibula respectively in preparation for a \#6 wood screw. Then the fibula was fastened to the tibia, as to maintain its natural anatomical position as best as could be approximated, with one 1-inch \#6 wood screw. Muscle was then dissected and removed from the Achilles tendon, leaving approximately $100 \mathrm{~mm}$ for clamping. Finally, each foot was placed in a jig and potted in a vertical position using a round mold and a low melting point bismuth alloy, see Figure 3-7. Once prepped, specimens were sprayed with a $0.9 \%$ saline solution for cleaning and tissue preservation and then refroze at $-20^{\circ} \mathrm{C}$ until testing. 


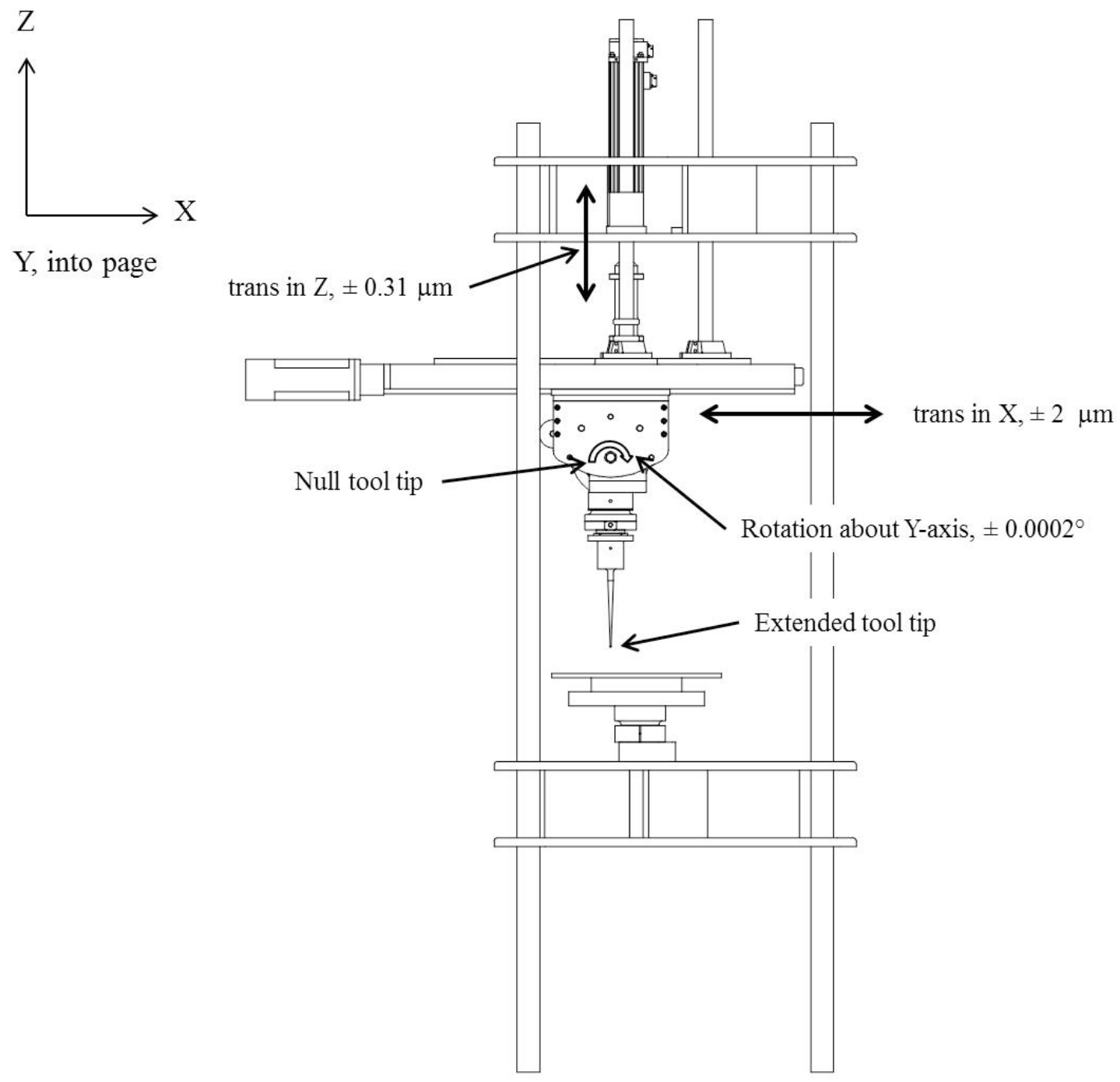

Figure 3-4. RTP after modifications.

Load capacity of the RTP is $890 \mathrm{~N}$ in the $\mathrm{X}$ axis, $4450 \mathrm{~N}$ in the $\mathrm{Z}$ axis, and $80 \mathrm{Nm}$ of continuous torque about the $\mathrm{Y}$ and $\mathrm{Z}$ axes. Also illustrated is the relative motion with resolutions and tool tips (NTT and ETT). 


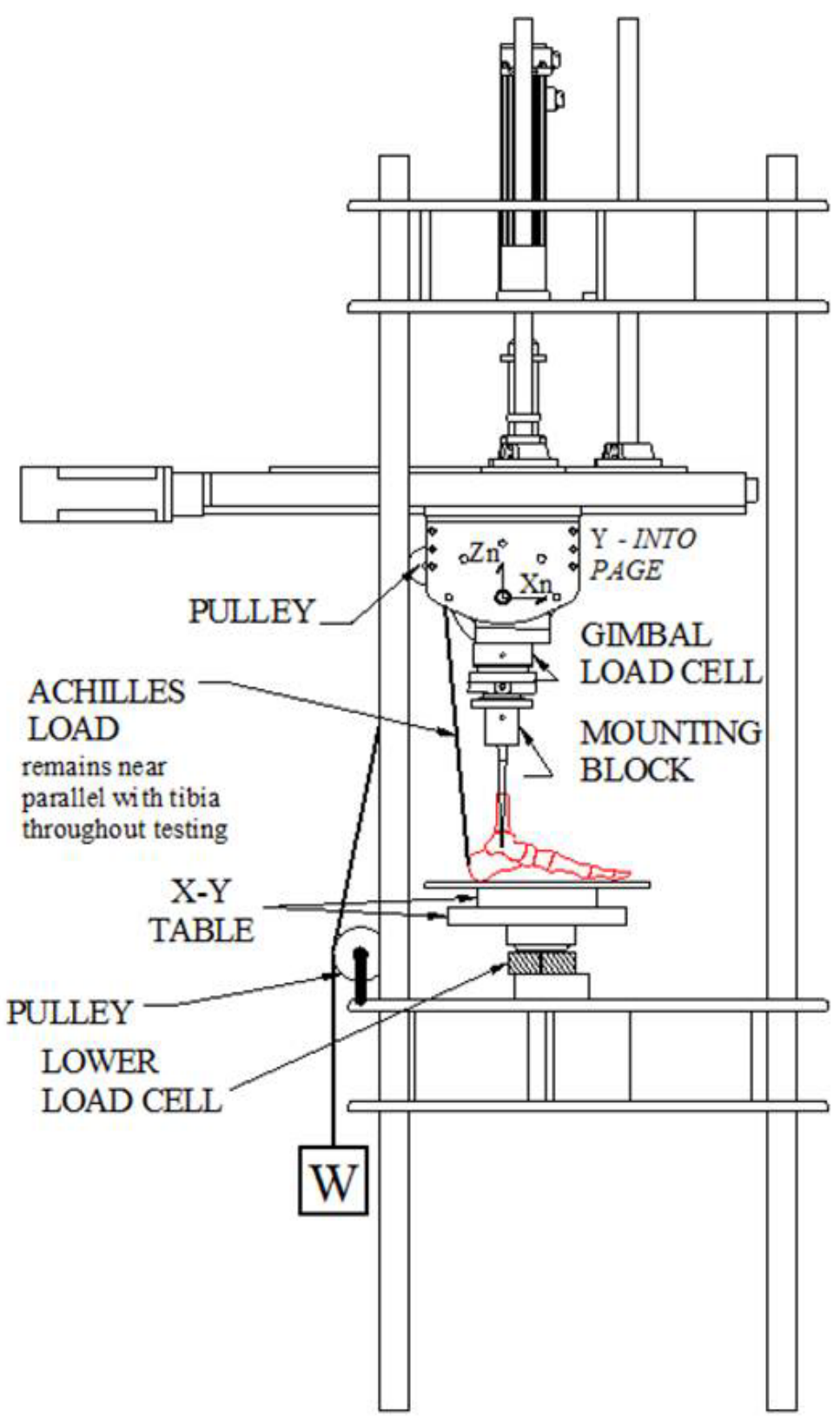

Figure 3-5. RTP coordinate system and test setup.

Illustrates coordinate system of the RTP and test setup of protocol. 


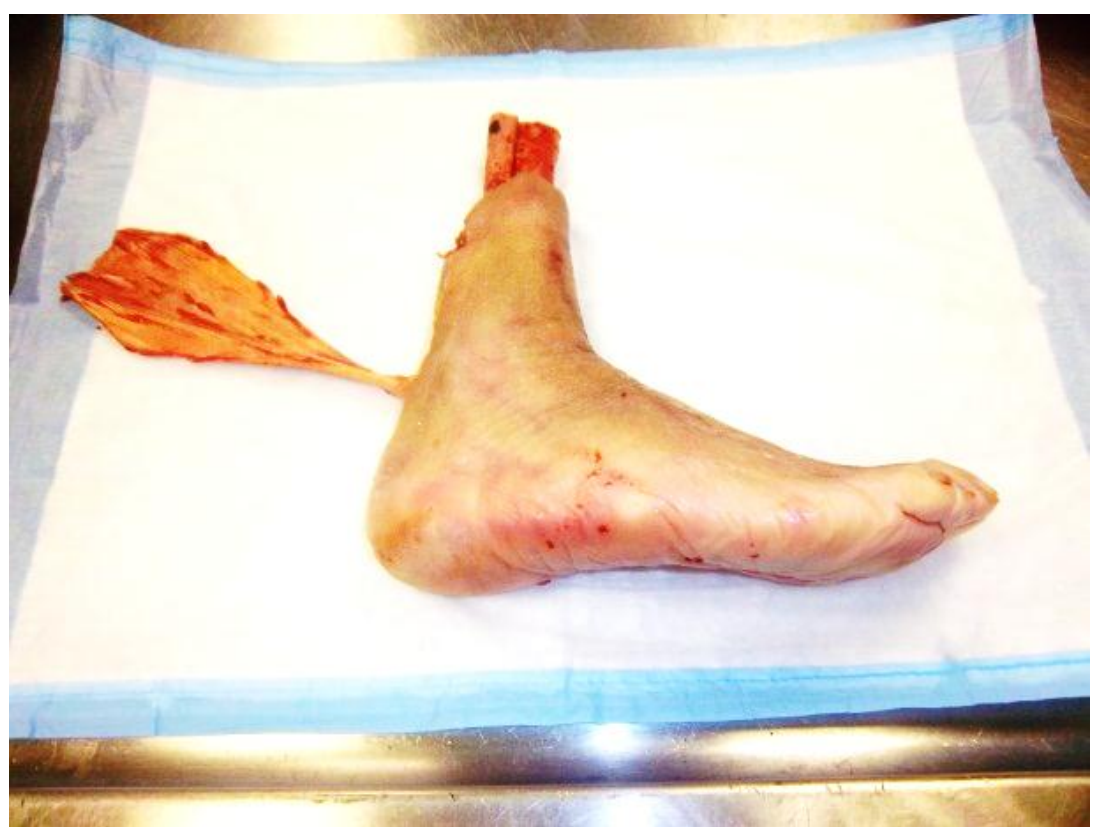

Figure 3-6. Dissecting tissue for testing.

Tissue dissected to expose tibia/fibula, Achilles tendon, and the fibula being secured to the tibia.

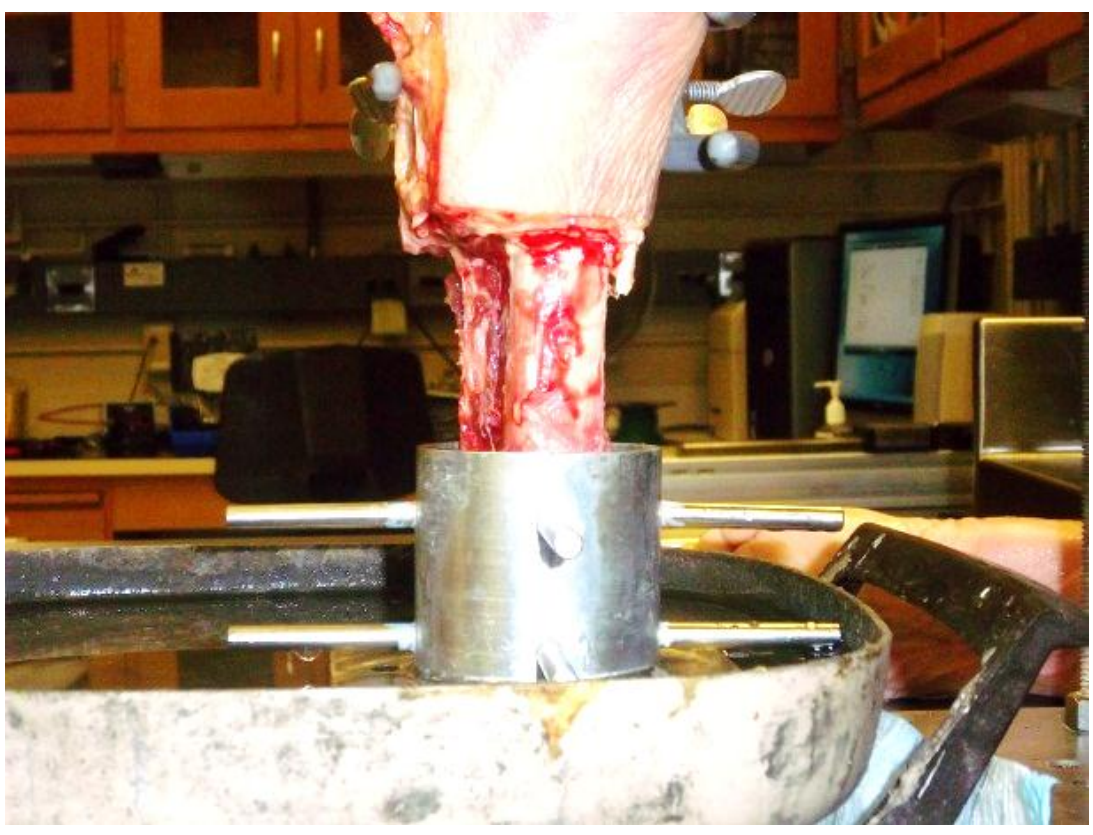

Figure 3-7. Potting specimen.

Specimen aligned vertically in potting mold. 


\section{CHAPTER 4. METHODS}

\section{Introduction}

Understanding the kinetics of a foot and ankle system is essential for developing medical devices or surgical techniques to treat certain foot pathologies or traumatic injuries. A testing protocol capable of measuring forces about the ankle and determining its IAR would aid in both design and evaluation of devices such as TARs where alignment and the ability to reproduce natural motion during gait are important. Using an in vitro testing approach to evaluate a foot and ankle system, a testing protocol was developed to determine the IAR pathway of the foot and ankle during dorsiflexion and plantar flexion at various loading conditions on the Achilles tendon.

Knowing how the IAR pathway changes following TARs, adding plates, inserting screws and foot injuries would be very advantageous in a clinical environment. The initial test would be conducted on specimens prior to surgical treatment or induced and serve as a baseline. Using an identical setup process, ensuring proper alignment, run specimens again and compare pathway against baseline run to see the effects of device alignment, loading, placement, or even surgical technique of installation.

\section{Specimen Preparation}

A sagittal plane was established for each specimen by bisecting the second metatarsal bone and the center line of the RTP, shown in Figure 4-1. This plane was aligned with the X-Z plane of the RTP and is the plane for evaluating the kinetics of the ankle. This method was utilized in other studies [6] to establish a testing plane. The potting material about the tibia and fibula is clamped securely by a mounting block to prevent any translation between it and the specimen, reference Figure 3-5. The mounting block is then rigidly connected to the RTP.

The specimen is mounted in the RTP with the tibia vertical with respect to the base plate to establish a neutral position at the ankle, refer to Figure 3-5. The position of the NTT at that instant became the origin for testing and any rotation from that point was measured as either dorsiflexion or plantar flexion. The upper load cell is aligned with the NTT and mounting block (clamps to potting material around tibia). The force frame of the upper load cell was transformed to read forces at the ETT.

A cable puller was then attached to the Achilles tendon. The cable puller was placed as low on the tendon as it would go and then a U-bolt was added at the base to increase clamping power, reference Figure 4-2. As confirmation of this clamping technique, $1500 \mathrm{~N}$ was applied and held for $60 \mathrm{sec}$ with no slippage witnessed.

The Achilles force was applied while the foot was in a heel-off condition, allowing the longitudinal arches to form. Use of an X-Y table allows this formation 


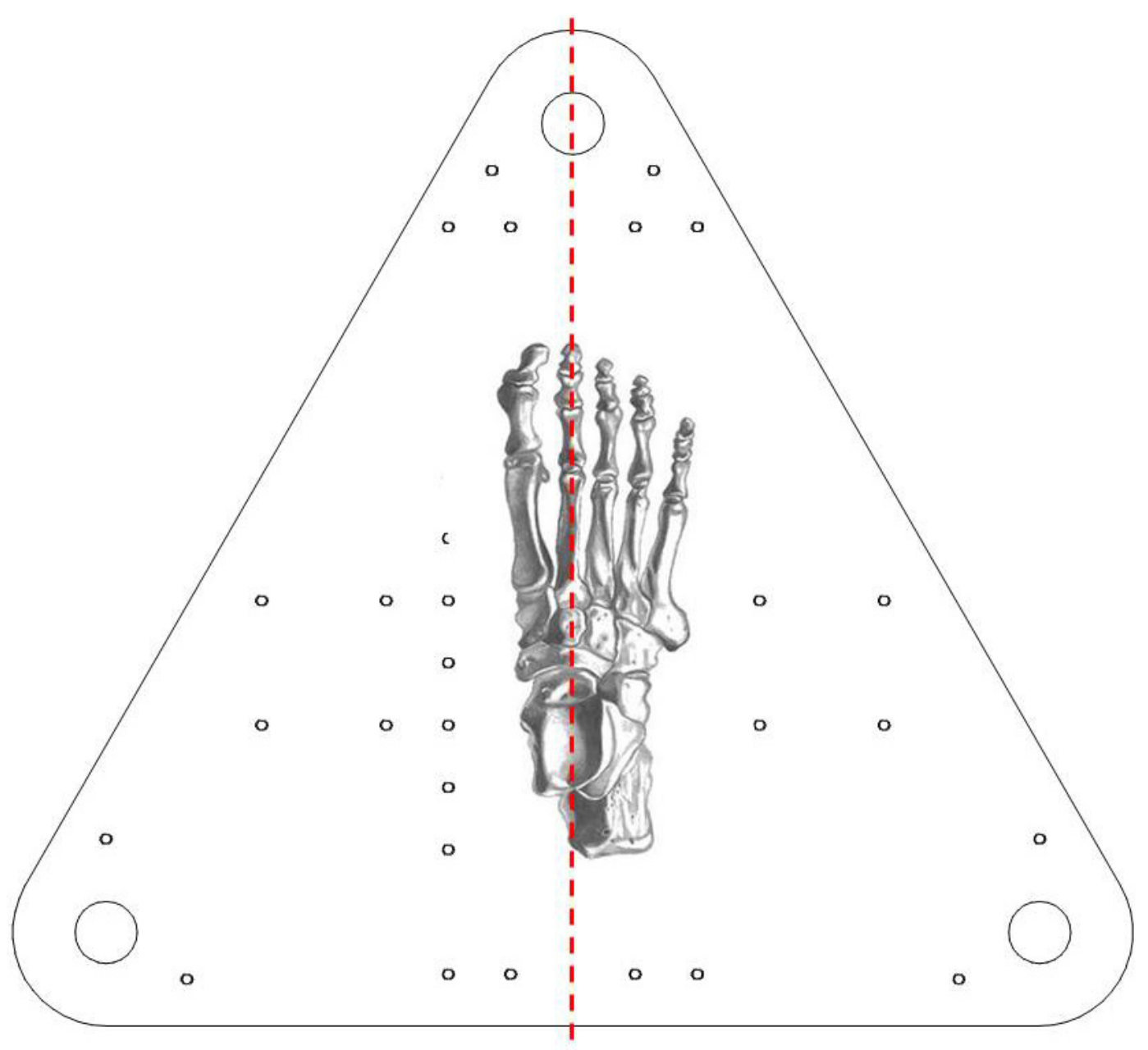

Figure 4-1. Specimen alignment.

The second metatarsal is aligned with the sagettal test plane for testing. The red line shows alignment for test setup. This method was established by Baxter et al. [Baxter,J. R.,T. A. Novack, et al. (2012). Ankle joint mechanics and foot proportions differ between human sprinters and non-sprinters. Proc Biol Sci. 22(1735):2018-24.]. Image of foot reprinted with permission from personal communication with Samantha Lobben. [Lobben, Samantha. (2008). Medical Illustration. Pencil on paper. http://www.samanthalobben.com.] 


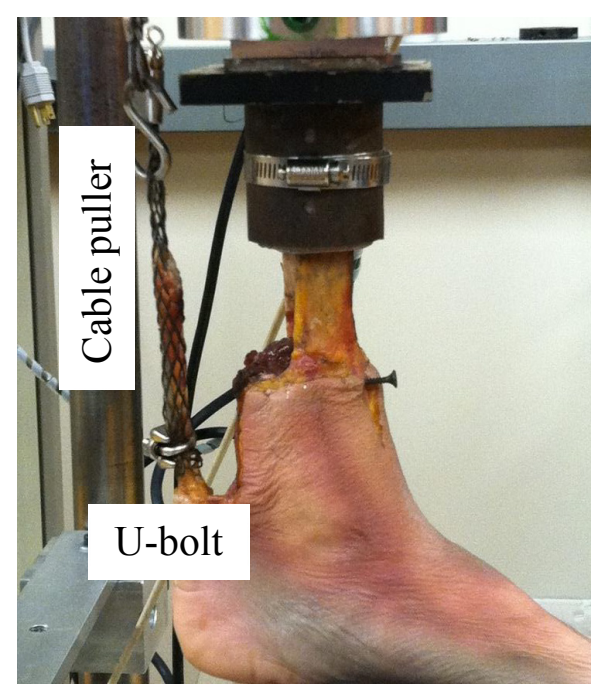

\section{Figure 4-2. Clamping of Achilles tendon.}

A standard cable puller in conjunction with a U-bolt provided a secure connection between the Achilles tendon and applied load.

without resistance. The foot is then lowered until heal makes contact and then the required tibia force is applied to meet specific test parameters at the ankle. The X-Y table was then locked to ensure ankle rotation without relative translation during testing.

\section{Testing Protocol}

The RTP was used to determine the IAR pathway of the ankle joint from a neutral position of $0^{\circ}$ to $10^{\circ}$ of dorsiflexion and again from a neutral position of 0 to $20^{\circ}$ of plantar flexion with and without a passive Achilles force. The passive force was created by hanging a series of calibrated weights from a cable attached to the Achilles tendon, simulating $25,50,75$, and $100 \%$ of the established vGRF. The vGRF used for testing was $355 \mathrm{~N}$. This was based on using $1 / 2$ the average male body weight (BW) of $710 \mathrm{~N}$. The tibia load generated at full BW exceeded the $800 \mathrm{~N}$ capacity of the upper load cell. At $1 / 2$ $\mathrm{BW}$ the theoretical tibia load is $710 \mathrm{~N}$ less weight of test specimen.

A custom software program was written to evaluate a "pure moment" condition when rotating a body about its instantaneous center while maintaining an Achilles force and linear force acting through the tibia center. This program measures off-axis forces in the $\mathrm{X}$ and $\mathrm{Z}$ axes and moves along the same axes in $0.25 \mathrm{~mm}$ steps (steps can be smaller) to reduce these loads to an acceptable value, typically $\pm 3 \mathrm{~N}$. The key incremental step in the program is illustrated in Figure 4-3.

First, a rotation is commanded about a point $(\Delta \theta)$, generally $0.5^{\circ}$. A moment is needed to generate this rotation $\left(\mathrm{M}_{\mathrm{apl}}\right.$, moment applied). As a result, forces build up in the 


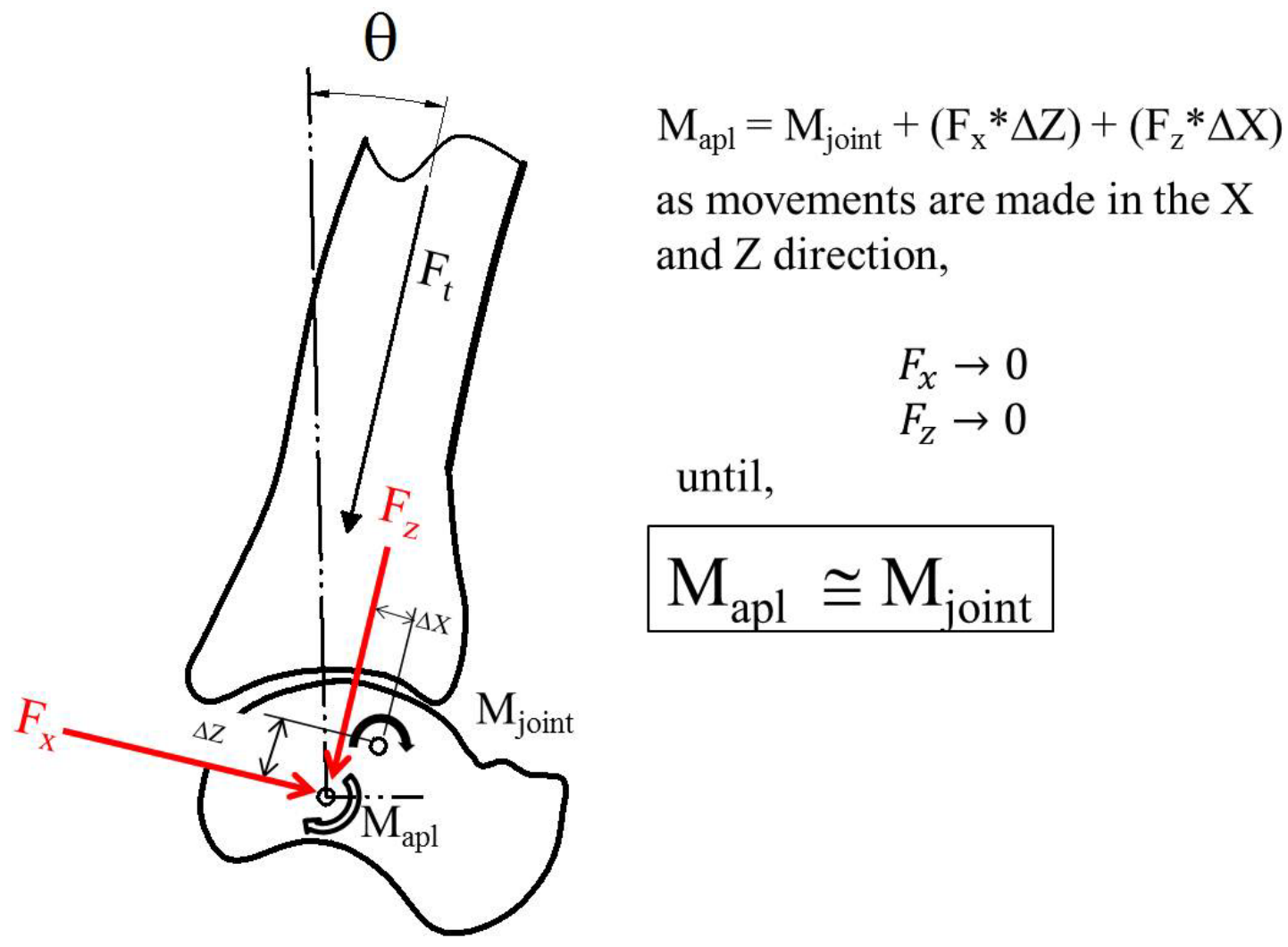

Figure 4-3. Incremental step from test protocol. 
$\mathrm{X}$ and $\mathrm{Z}$ axes' about the initial point of rotation, indicating the center of rotation is out of position for the movement. This point is then repositioned by taking small incremental steps of $0.25 \mathrm{~mm}$ in the $\mathrm{X}$ and $\mathrm{Z}$ axes to eliminate the off-axis loads, or the effects thereof, so you are left with the actual moment $\left(\mathrm{M}_{\text {joint }}\right)$ required to rotate the ankle complex about its true center. That point is stored and identified as the lowest energy point or point that requires the least amount of torque to rotate $\Delta \theta$. This now reduces the moment generated by the off-axis loads so the only moment remaining is the actual moment required to rotate the joint, allowing soft tissue and the articulating surface define this rotation. This process is repeated until the full amount of flexion or extension is achieved.

However, before this step an initial rotational point has to be established. This is accomplished by measuring the distance from the center point on the top surface of the potting material to the perceived center of the ankle, approximated as the center of the talus dome. Offset distances in the $\mathrm{X}$ and $\mathrm{Z}$ directions can be accounted for with respect to this point. With radiographic images of each specimen these measurements are made via Image-J software, refer to Figure 4-4.

The stored data points from the custom program are saved in a text file unique to the test ran. The data contains complete positional data (X, Y, Z, and $\theta$ ) for the NTT and the ETT and forces $\left(F_{x}, F_{y}, F_{z}, M_{x}, M_{y}\right.$, and $\left.M_{z}\right)$ measured about the ETT, reference Figure 4-5.

Crisco et al [20] introduced a method for determining the IAR of a rotating mechanism using starting point, ending point and rotational data of one point on a rigid body. This method is based on Reuleaux's principle of intersecting bisectors, refer to Figure 4-6.

In Crisco's case his computations were more involved because he had to calculate the angle of rotation first and then apply his equations. Since we have a very accurate measure of the angle rotated and translations in $\mathrm{X}$ and $\mathrm{Z}$ axes per incremental step, we were able to apply the following equations directly:

$$
\begin{array}{ll}
\mathbf{X}_{\mathrm{iar}}=\frac{\mathbf{1}}{\mathbf{2}}\left(\mathbf{X}_{\mathbf{1}}+\mathbf{X}_{2}\right)+\frac{\left(\mathbf{Z}_{1}-\mathbf{Z}_{2}\right) * \sin \boldsymbol{\theta}}{2 *(1-\cos \theta)} & \text { Eq. } 4-1 \\
\mathbf{Z}_{\text {iar }}=\frac{\mathbf{1}}{\mathbf{2}}\left(\mathbf{Z}_{1}+\mathbf{Z}_{2}\right)-\frac{\left(\mathbf{X}_{1}-\mathbf{X}_{2}\right) * \sin \boldsymbol{\theta}}{2 *(1-\cos \theta)} & \text { Eq. } 4-2
\end{array}
$$




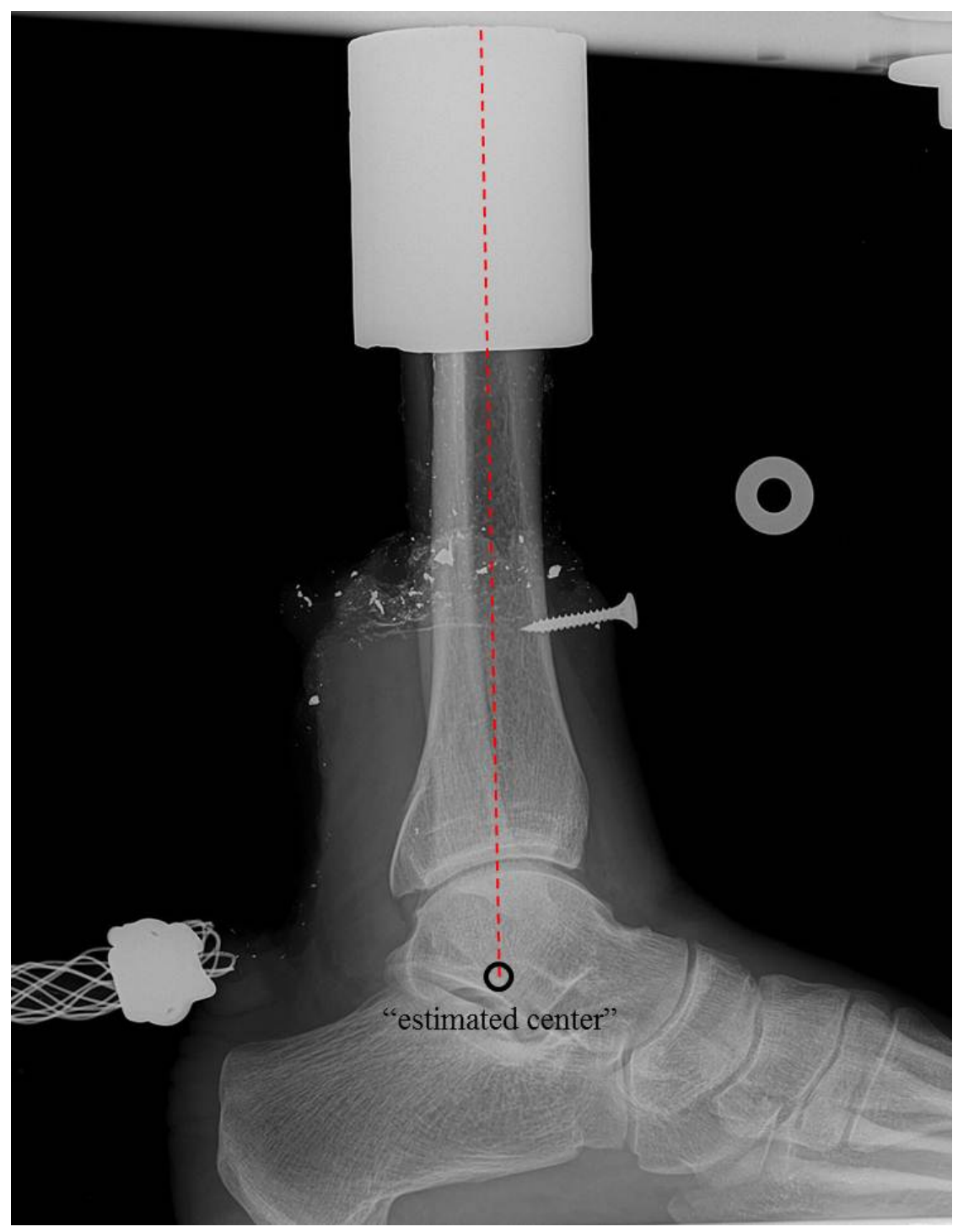

Figure 4-4. Initial center of rotation, needed for protocol.

Approximate the initial center rotation at the center of the talus, based on the talus dome, as shown here (scaled radiograph). Next measure down from the center of the potting material to the estimated center of the talus (red line). $17 \mathrm{~mm}$ (thickness of mounting plate) is added to this measurement and establishes the initial distance to the common reference plane, shown in Appendix B, and becomes the initial point of rotation. It is also the point that the NTT and force frame are translated to and data collected about. 


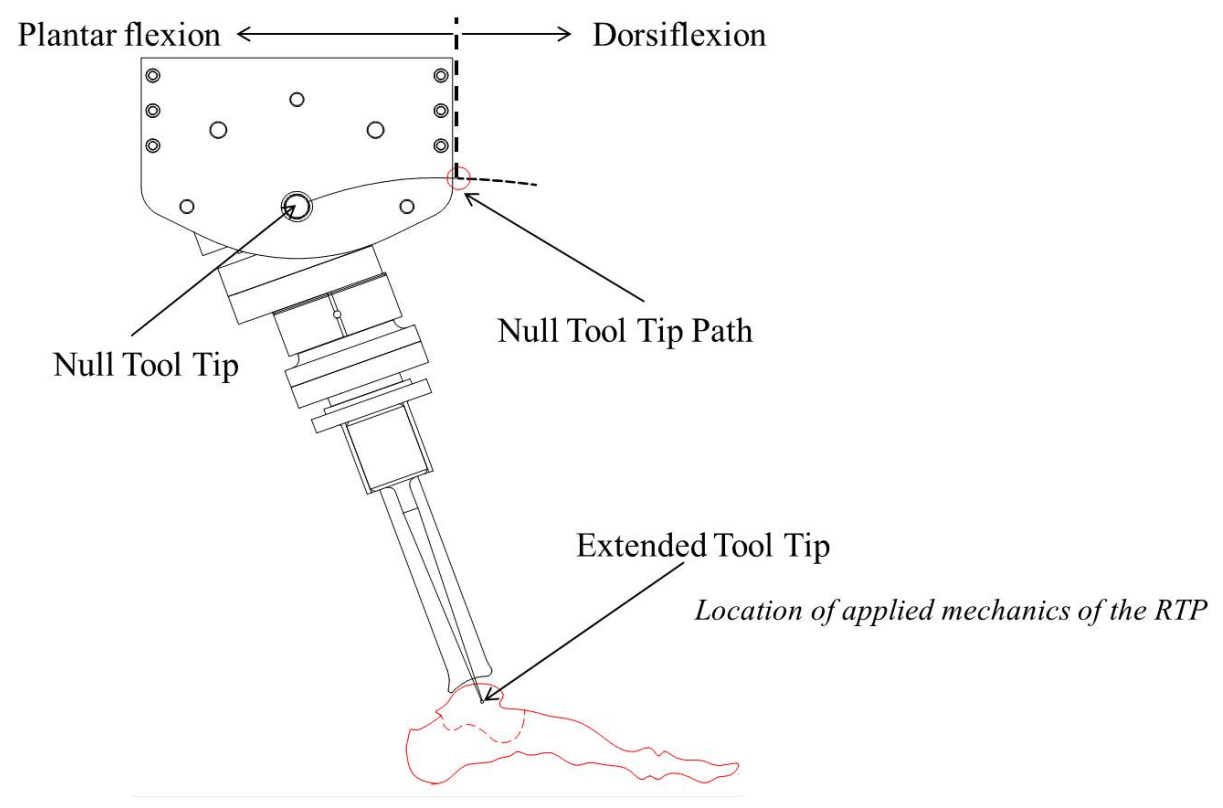

Figure 4-5. Null tool tip path during testing.

Illustrates shape and direction of the NTT path used to calculate the IAR, also, reiterates the ETT as the point of applied mechanics during testing.

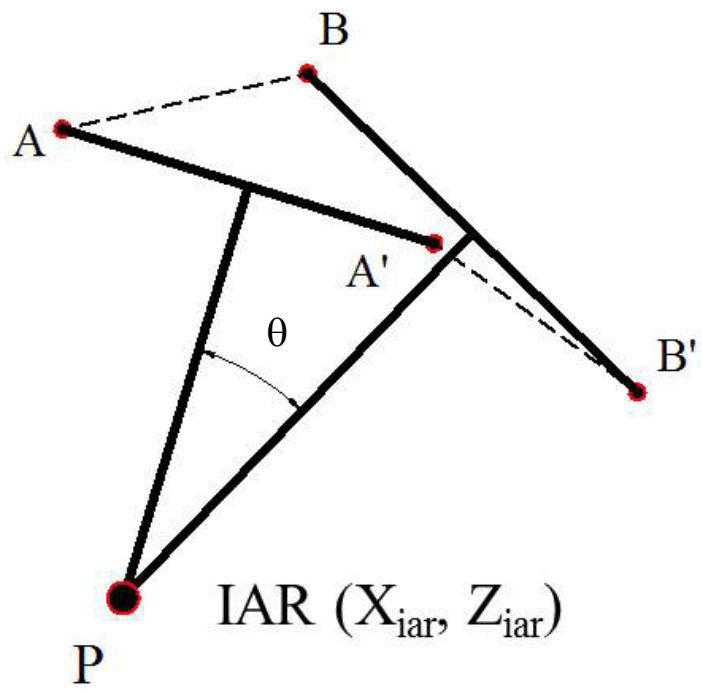

Figure 4-6. IAR established by intersecting perpendicular bisectors.

Reduction of Crisco equations requires only one point on a rigid body be tracked:

$\mathrm{A}(\mathrm{Xn} 1, \mathrm{Zn} 1) \& \mathrm{~A}^{\prime}(\mathrm{Xn} 2, \mathrm{Zn} 2)$ represent starting and ending points on a rigid body that has undergone rotational and linear translation and $\mathrm{P}$ (Xiar, Ziar) is the instantaneous axis of rotation for that movement. 


\section{Data Management and Statistical Analysis}

\section{Repeatability}

A repeatability study was developed to evaluate how consistent the RTP is when testing the same specimen. The protocol was varied slightly so that the RTP and protocol can be evaluated without the effects of setup variance. For this test, the specimen was mounted to the RTP and Achilles loaded just as before. The specimen was cycled five times consecutively without raising the heel and reapplying the Achilles load between runs and the IAR pathway calculated. This was done for both dorsiflexion and plantar flexion. Since tissue relaxation is still an issue the last three runs of each test were used to evaluate repeatability of this protocol. This minimized relaxation effects in our data.

A sixth order polynomial curve fit of each data set was conducted. This established the best fit. A mean curve was created from pooled data for each group. Then a one-way ANOVA utilizing a Bonferroni post hoc test to compare coefficients of the curve fits was conducted with GraphPad statistical software package (GraphPad Software, La Jolla, CA). Each curve in their group was compared to the mean curve and then to each other. As a secondary check the means of each curve were analyzed in the same manner of a one-way ANOVA, however, this time an S\&K post hoc test was used to compare means.

\section{Protocol Error}

As a means to explore the error in this technique, a 4-bar linkage model was created and analyzed in Working Model 2D (WM). A point was established on a rigid body in the model and the IAR pathway for that rigid body was recorded under the prescribed motion. Rotational angle and positional data were also recorded for the point. This gave us data for starting and ending point locations along with rotational increments. This data was then used in our normal data processing method of smoothing before calculating the IAR. Then theoretical data was compared to simulation data from WM.

The data was then analyzed by comparing the errors of the data sets. Error data were averaged and standard deviation calculated to evaluate the variance in the protocol. This in return establishes an accuracy level of the protocol.

\section{Effects of Achilles Load}

A means to apply a passive load on the Achilles tendon was established to study its effect on the kinematic profile of the ankle. The testing conditions are listed in Table 4-1. The IAR pathway at each condition was calculated and graphed. Then a one-way ANOVA utilizing a Bonferroni post hoc test to compare coefficients of the curves and a S\&K post hoc to compare the means was conducted. 
Table 4-1. Achilles loading conditions.

\begin{tabular}{cccc}
\hline \% vGRF & vGRF & $\mathbf{F}_{\mathbf{a}}$ & $\mathbf{F}_{\mathbf{t}}$ \\
\hline 0 & 355 & 5 & 5 \\
25 & 355 & 89 & 404 \\
50 & 355 & 178 & 493 \\
75 & 355 & 266 & 581 \\
100 & 355 & 355 & 670 \\
\hline
\end{tabular}

The Achilles tendon was loaded as a percentage of the vertical ground reaction force acting on the foot. All force values listed are in Newtons. Refer to Figure 2-3 (FBD) for loading relationships.

\section{Smoothing Null Tool Tip Data}

Since our data points were taken statically and with force tolerances in the custom program, the NTT paths seemed jagged, refer to Figure 4-7, with respect to rotation and introduced perturbations into our equations resulting in non-uniform results and often producing what appeared to be outliers. Crisco et al [20] discussed sensitivity to noise in data and the effects it has on the results. It was similar to what we experienced here. To overcome this we smoothed the $X_{n}$ and $Z_{n}$ data with respect to $\theta$ for a better relationship between data points. It was determined that a second order polynomial curve fit was best because it had equivalent or better variance than higher order polynomial curve fits, reference Appendix $\mathrm{C}$ for example. The IAR was then calculated with the smoothed data for all testing conditions; refer to Table 4-1 for testing conditions.

\section{Normalizing Final Data}

In order to compare results from different specimen, a generic model of the tibia and talus was created; refer to Figure 4-8. The potting material is rigidly connected to the RTP and the distance from the NTT to the top mounting block is known. Radiographs of each specimen are taken in a medial view, clearly displaying the tibia and talus relationship, and scaled accordingly so the IAR can be graphed with respect to the NTT and talus. The widest point of the distal end of tibia is used as a common landmark and each specimen data set is scaled to this baseline measurement. 

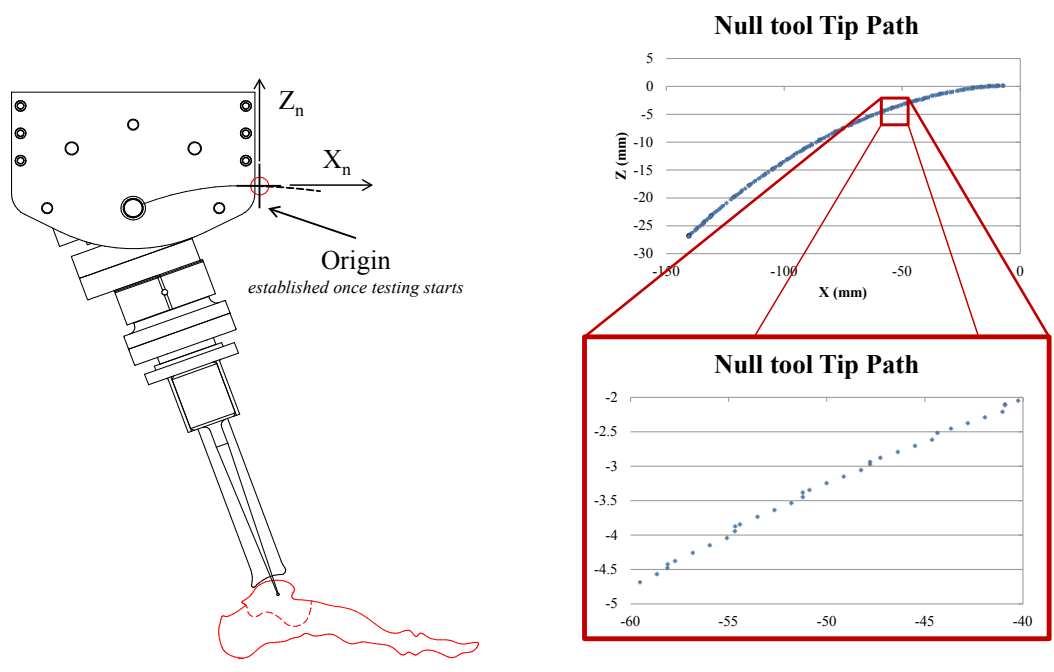

Figure 4-7. Null tool tip data before smoothing process.

From a high level view the NTT path appears relatively smooth, however, zooming in reveals small abrupt changes in direction that had severe effects on the IAR calculations.

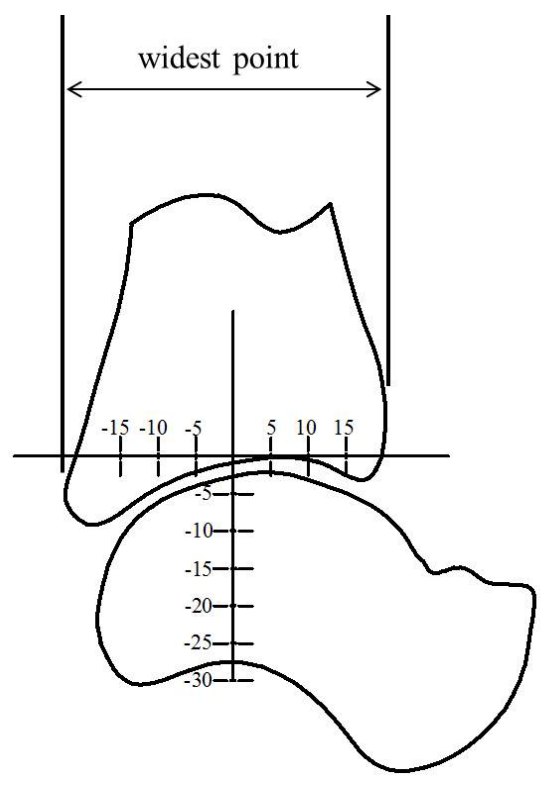

Figure 4-8. Normalized chart for plotting various IAR results.

Using the radiograph of a specimen in a neutral position a horizontal line tangent to the articular surface of the tibia was drawn. Then a vertical line from the center of the potting material down through the talus was drawn. Where those lines intersected became the origin for this generic model. 


\section{CHAPTER 5. RESULTS}

\section{Repeatability}

After processing the data the results were compiled and charted. The curves for dorsiflexion and plantar flexion appeared very similar. Figure 5-1 illustrates the close proximity of these curves. A statistical analysis was performed to determine if the curves in each group were significantly different via Bonferroni test. Each curve in their group was compared to the mean curve and then to each other. The results concluded that there was no significant difference $(\mathrm{P}<0.05)$ between the curves in each group. Also, an analysis was performed on the means via $\mathrm{S} \& \mathrm{~K}$ test and again no significant difference $(\mathrm{P}<0.05)$ in the data.

\section{Protocol Error}

Using Working Model 2D, a simulation software package, a 4-bar linkage model was created to define a known IAR pathway and tool tip path (similar to the null tool tip in this study). Complete positional data for the tool tip (a point established on a rigid body) was established to include translational and rotational data. The tool tip data was processed through the IAR protocol to determine its kinematic profile and compare it to the actual profile generated in Working Model 2D. Shown in Figure 5-2, the curves are nearly identical. Error data shown in Table 5-1 was conducted based on analyzing residual data.
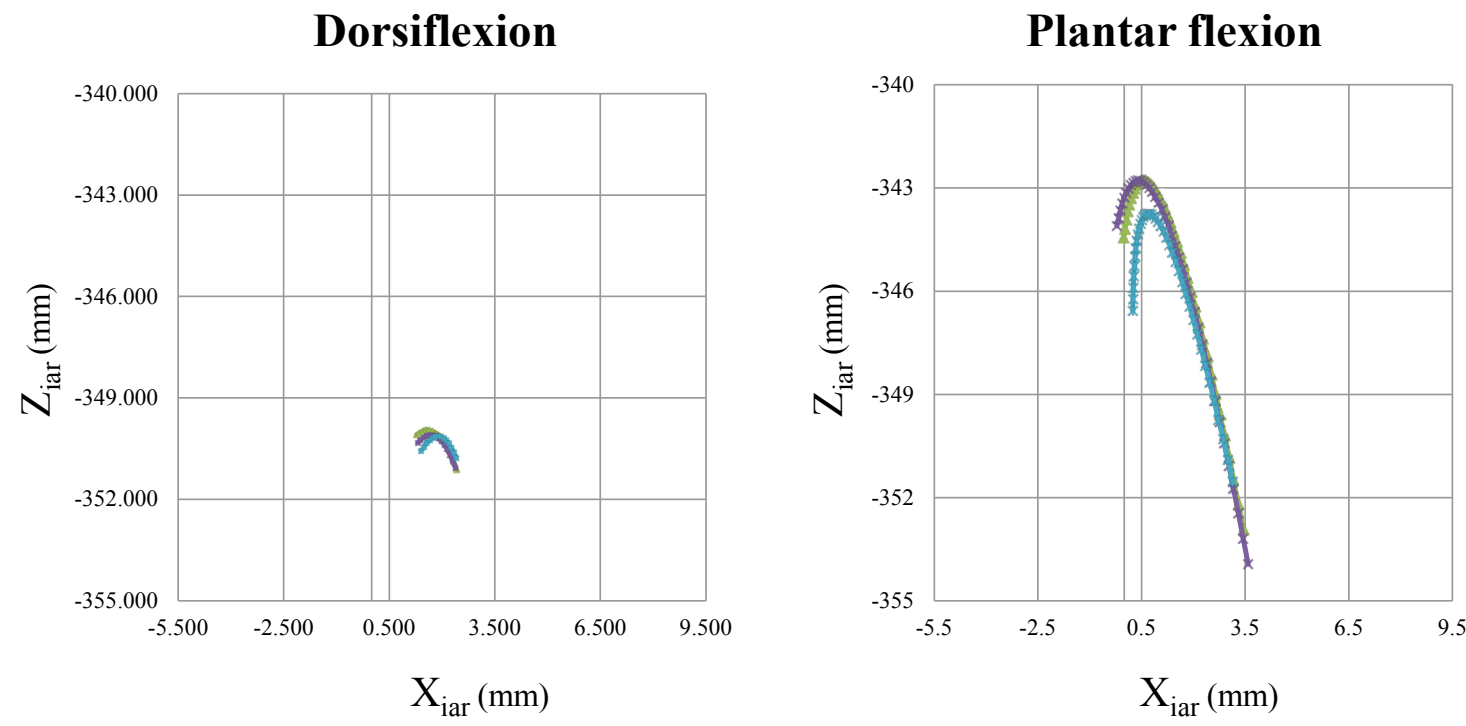

Figure 5-1. IAR curves used to evaluate repeatability of RTP and protocol. 


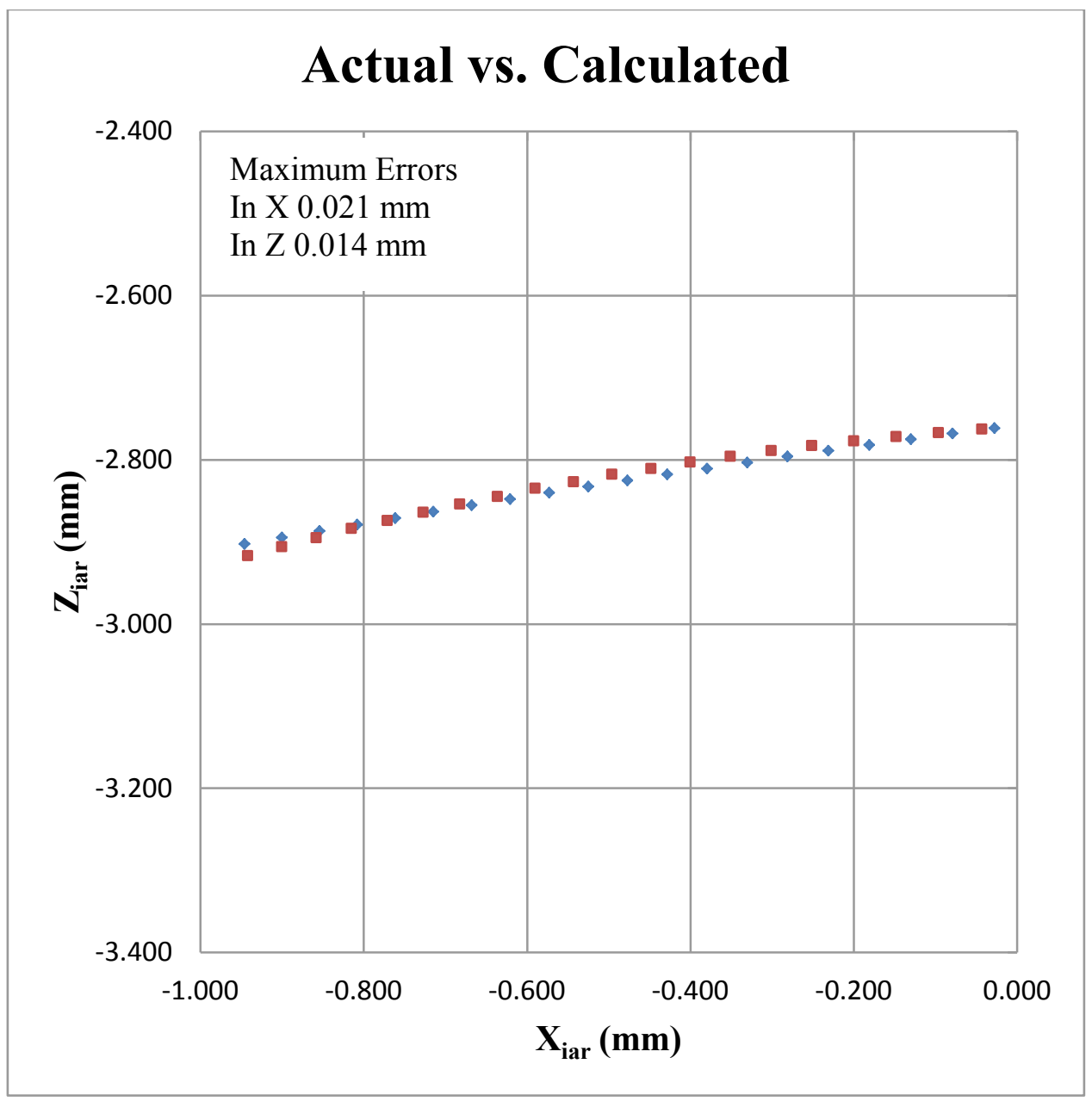

Figure 5-2. IAR data curves.

The red curve was actual data for a 4-bar linkage model and the blue curve calculated data via protocol. Here a rigid body of concern rotated from $0^{\circ}$ to $25^{\circ}$ at $1.2^{\circ}$ increments (limited by simulation data).

Table 5-1. Protocol error induced based on angular step size.

\begin{tabular}{cccc}
\hline Error in & Range (mm) & Avg (mm) & Stdev (mm) \\
\hline $\mathrm{X}_{\mathrm{iar}}$ & 0.028 & -0.013 & 0.008 \\
$\mathrm{Z}_{\mathrm{iar}}$ & 0.026 & 0.000 & 0.008 \\
\hline
\end{tabular}

These results are based on simulation data where the rigid body of concern rotated from $0^{\circ}$ to $25^{\circ}$ at $1.2^{\circ}$ increments (limitation of simulation data). This error is dependent on the size of the rotational increment of the data set. The smaller the rotational increment the smaller the error. 


\section{Effects of Achilles Load}

The results for each specimen were charted individually for dorsiflexion and plantar flexion as shown in Figure 5-2. The data along with the analysis revealed a smooth IAR pathway. Results for dorsiflexion indicate that different features may be governing rotation at various loading conditions. Each IAR pathway is noticeably different from each other, see Figure 5-3.

When comparing dorsiflexion data between specimens, pathways at $50 \%$ of vGRF were similar. For that reason specimen 1 and 2 were compared at $50 \%$ of vGRF in both dorsiflexion and plantar flexion for uniformity, refer to Figure 5-4. These results are consistent with those of Leardini [6] and Baxter [14], refer to Figures 5-5 and 5-6. Both show the center of rotation within the talus. Also, when comparing the IAR of a single test specimen with and without an Achilles load there is a stark difference in the results. 

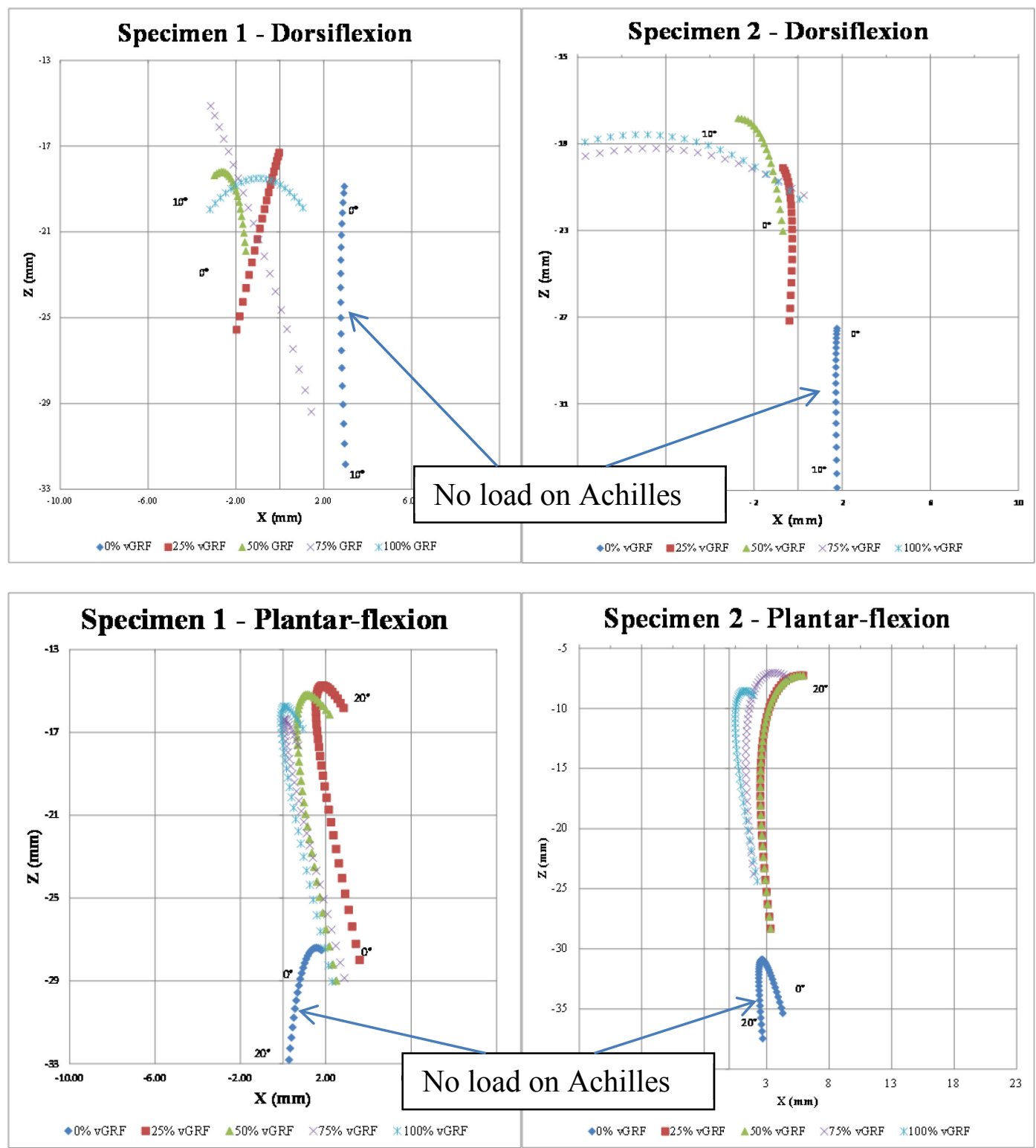

Figure 5-3. IAR data for specimens 1 and 2 at various loads on Achilles At no load conditions the IAR pathway moves in an inverse direction with respect to IAR pathways at loaded conditions. 

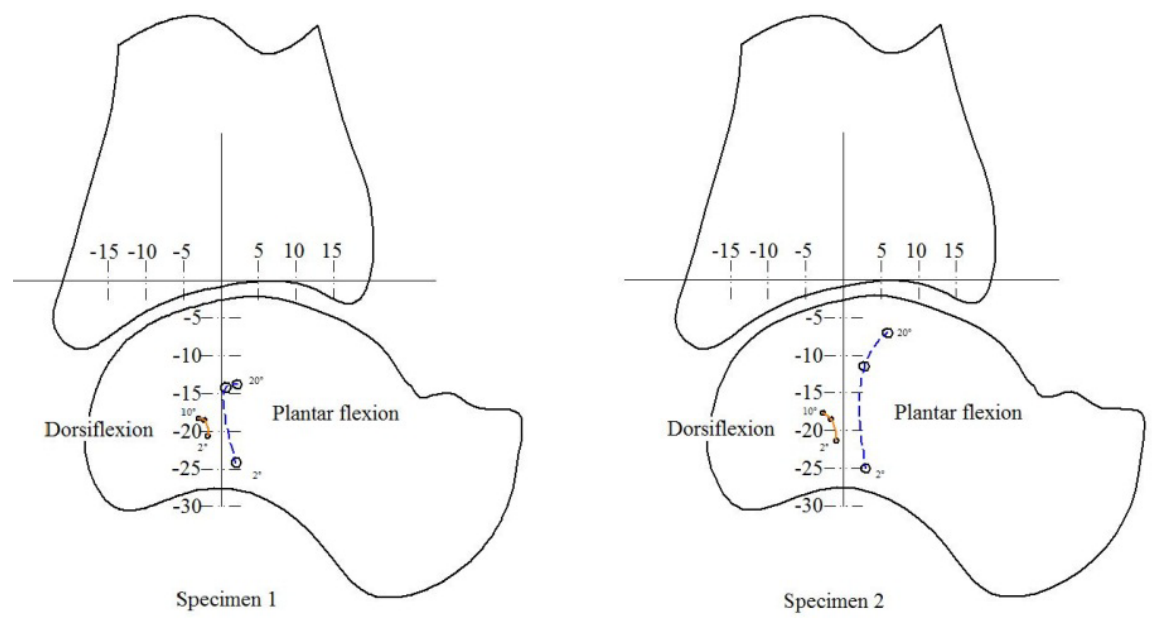

Figure 5-4. IAR results for specimen $1 \& 2$.

These results are at $50 \% \mathrm{VGRF}$ and range from $2^{\circ}$ to $10^{\circ}$ dorsiflexion and $2^{\circ}$ to $20^{\circ}$ plantar flexion.

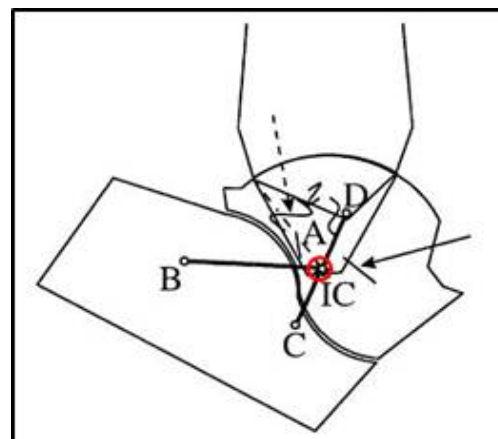

(a)

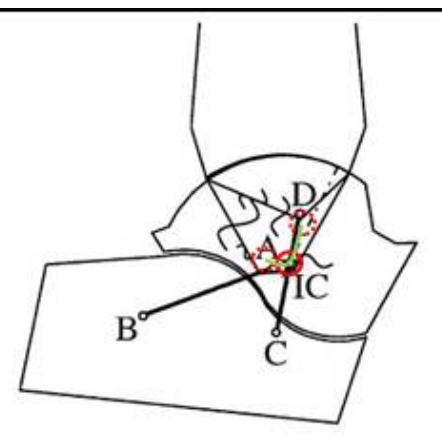

(b)

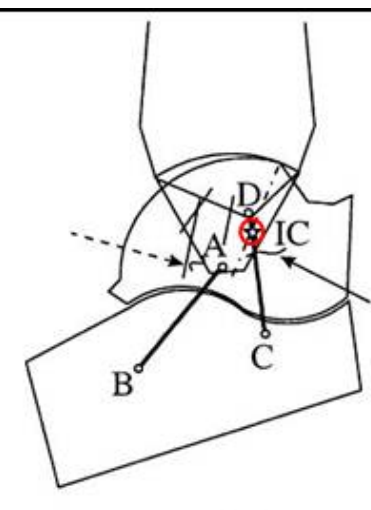

(c)

Figure 5-5. Image from Leardini et al showing IAR path.

(a) plantar flex position. (b) neutral position. (c) dorsiflexion position. Leardini et al model the foot and ankle as a four bar linkage system. Using four bar linkage theory, were links $\mathrm{AB}$ and $\mathrm{CD}$ intersect (red circle) defines the instantaneous axis of rotation for link AD (tibia with respect to the talus). Looking at (b) the outward dashed circles are the end points for the IAR path. Source: Reprinted with permission. Leardini, A., J. J. O'Connor, et al. (2004). Mobility of the human ankle and the design of total ankle replacement. Clin Orthop Relat Res(424): 39-46. 


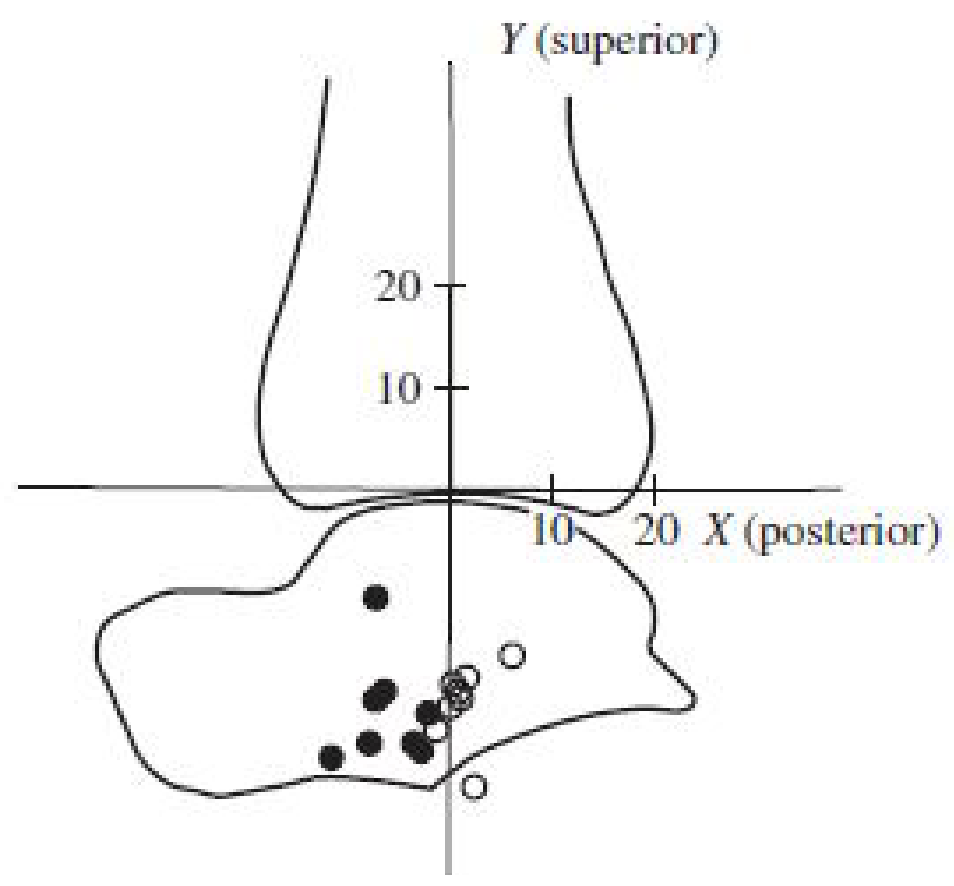

Figure 5-6. Image from Baxter et al showing single point approximation.

Here the center of rotation for both dorsiflexion and plantar flexion is approximated as a single point of rotation. These results are from in vivo studies, applying a Reuleaux-like method to magnetic resonance images when the foot is rotated from $15^{\circ}$ dorsiflexion, neutral $0^{\circ}$, and $15^{\circ}$ plantar flexion. Source: Reprinted with permission. Baxter,J. R., T. A. Novack, et al. (2012). Ankle joint mechanics and foot proportions differ between human sprinters and non-sprinters. Proc Biol Sci. 22(1735):2018-24. 


\section{CHAPTER 6. AN INNOVATIVE TESTING PROTOCOL FOR THE FOOT AND ANKLE COMPLEX}

\section{Introduction}

Surgical treatment of the ankle joint is problematic due to its inherent complexity. For severe ankle pain, resulting from disease or trauma, common medical procedures are total ankle replacements (TAR) or ankle arthrodesis. Short comings of ankle arthrodesis are its impact on gait mechanics and transference non-physiological forces to adjacent joints. For TARs major causes of failure are misalignment of implant, loosening of components (largely tibial components), and improper mechanics (implant did not behave like an ankle joint), as reported by Gougoulias et al [3]. It is critical that the kinematics of the ankle complex be accurately studied. is critical and having robust test platform is key to this.

Few testing platforms can evaluate lower limb kinetics $[8,9,10,11,12]$, and of those the instantaneous axis of rotation (IAR) is approximated or forced to follow a set IAR pathway that is not its own. Even with strong evidence that the ankle behaves according to a system with an instantaneous axis, many still approximate a single point of rotation for the ankle during rotation [13] or use overly simplified techniques as graphically tracking landmarks over large angles [14] on an X-ray. Hence, there is scope for development of a new, validated testing platform.

The objective going forward was to develop robotic testing platform (RTP) and testing protocol to evaluate foot and ankle mechanics with and without a passive Achilles force. The testing protocol is to be based on a pure moment test to establish the "path of least resistance" or lowest energy state to rotate the tibia about the ankle complex. Then study the kinetics of the ankle complex with and without an Achilles load and compare the results.

\section{Mechanics of Foot and Ankle}

There are three motions in which the foot moves, each with its own axis and plane of motion, dorsiflexion-plantar flexion, abduction-adduction, and eversion-inversion. The foot is comprised of three arches, medial longitudinal, lateral longitudinal, and transverse, to help transfer high loads during normal active loading. Figure 6-1 illustrates loading

conditions of foot and ankle. In our approach we passively loaded the Achilles tendon as a percentage of the vGRF acting on the foot and ankle to activate the longitudinal arches. We assumed the average body weight to be $712 \mathrm{~N}$ and set the vGRF at half body weight $(356 \mathrm{~N})$.

Gait data presented by Winter [18] describes the dynamic loading characteristics of the ankle. This data was established via force plate measurements and tracking optical markers. With this data a rigid body analysis can be used to evaluate the effects of a force 

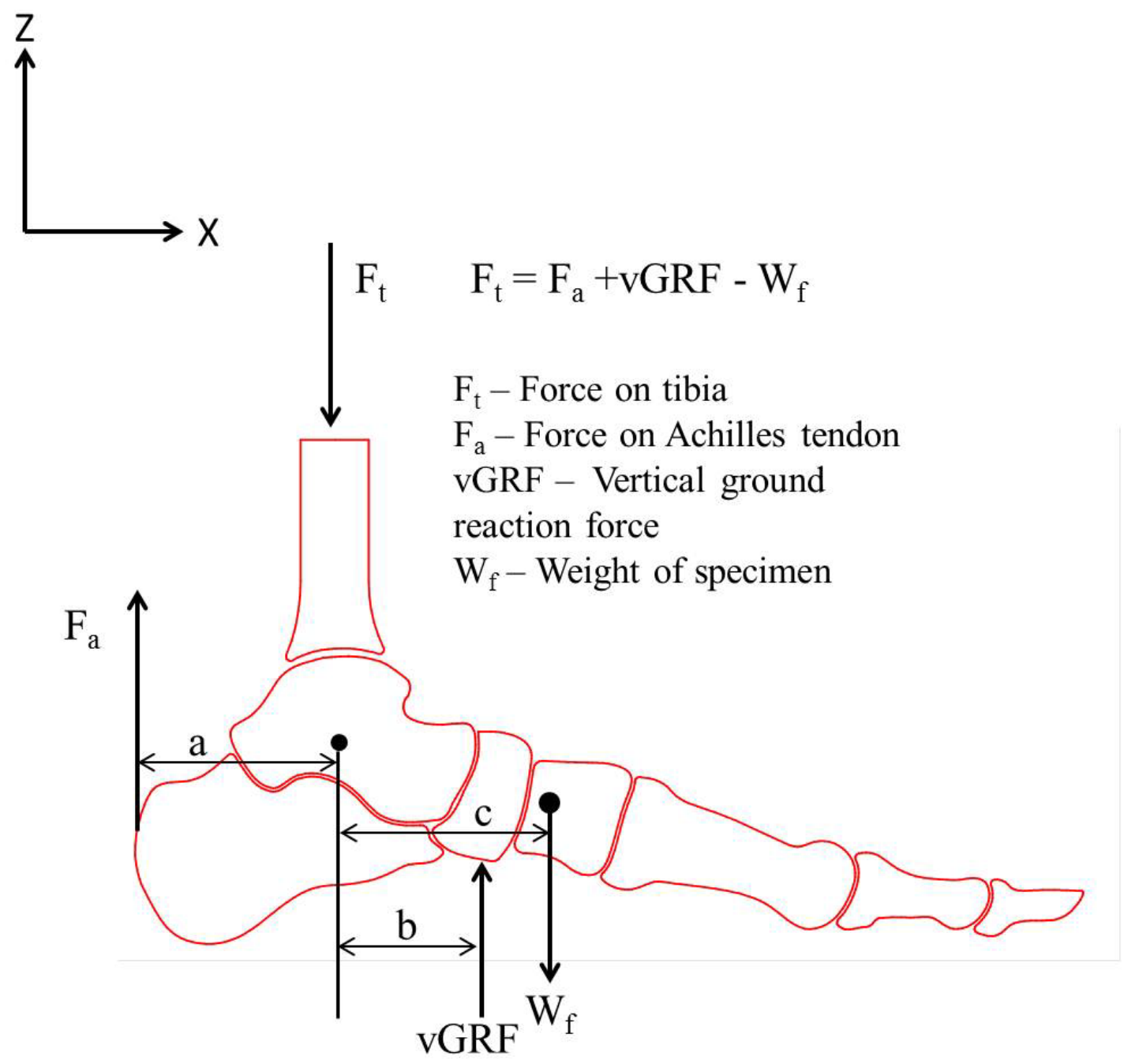

Figure 6-1. Free body diagram of foot and ankle complex.

The ankle is shown in a neutral position with the tibia normal to the ground. Also, the longitudinal arch is shown, including the bones that form the arch. 
acting in the direction of the Achilles tendon $\left(\mathrm{F}_{\mathrm{a}}\right)$ and a vertical ground reaction force (vGRF) has on ankle kinetics.

\section{Methods and Materials}

\section{Specimen}

A matched pair of feet (male, age 50) was used to evaluate the abilities of the RTP in calculating the IAR of the ankle under a given vGRF and an Achilles load at 25, 50,75 , and $100 \%$ of vGRF. Specimens were cut below the knee to be approximately 254 $\mathrm{mm}$ in height. Muscle and soft tissue was dissected and removed to expose $76.2 \mathrm{~mm}$ of the tibia and fibula at the top of each specimen. Clearance and engagement holes were predrilled into the tibia and fibula respectively in preparation for a \#6 wood screw. Then the fibula was fastened to the tibia, as to maintain its natural anatomical position as best as could be approximated, with 1 one inch \#6 wood screw. Muscle was then dissected and removed from the Achilles tendon, leaving approximately $100 \mathrm{~mm}$ for clamping. Finally, each foot was placed in a jig and potted in a vertical position using a round mold and a low melting point bismuth alloy.

\section{Testing Platform}

The RTP has 4 degrees of freedom (DOF) and monitors two 6-axis load cells, shown in Figure 6-2. The RTP has a positional accuracy of $2 \mu \mathrm{m}$ in the X-axis and 0.31 $\mu \mathrm{m}$ in the Z-axis [19]. The rotary actuator used to rotate about ( $\mathrm{Y}$ and $\mathrm{Z}$ axes) has a resolution of $0.008^{\circ}$.

The load cells used in the RTP measure the following forces: $F_{x}, F_{y}, F_{z}, M_{x}, M_{y}$, and $\mathrm{M}_{\mathrm{z}}$. They can be used together or one at a time based on the needs of testing. The resolution for gimbal and lower load cells are $0.4 \mathrm{~N}$ and $1.5 \mathrm{~N}$ respectively.

With the positional accuracy of the RTP the null tool tip (NTT) is recorded verses time or step count to within $2 \mu \mathrm{m}$. Since the extended tool tip (ETT) is rigidly connected to the NTT its exact position is recorded in the same format and same accuracy. Refer to Figure 6-3. The force frame of the upper load cell is transformed to the ETT so forces about the ETT are measured and recorded.

\section{Mounting Specimens for Testing}

A sagittal plane was established for each specimen by bisecting the second metatarsal bone and the Achilles tendon. This plane was aligned with the $\mathrm{X}-\mathrm{Z}$ plane of the RTP and is the plane for evaluating the kinetics of the ankle. The potting material about the tibia and fibula is clamped securely by a mounting block to prevent any 


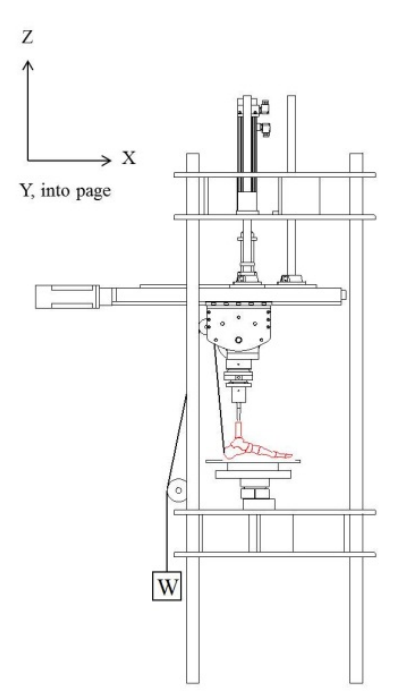

A

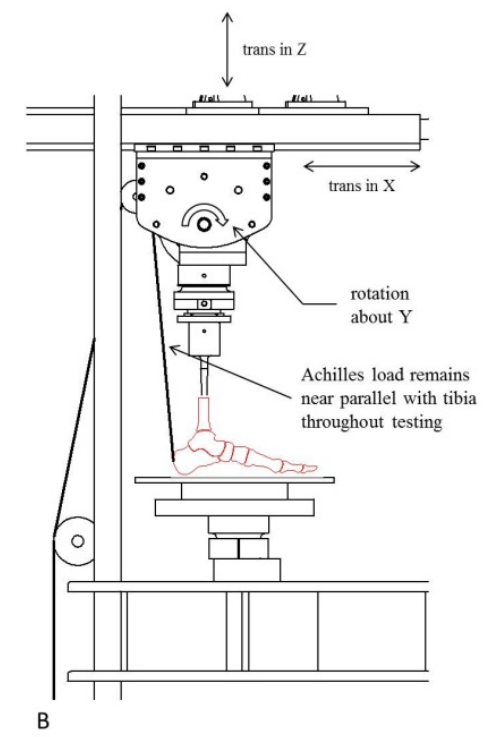

Figure 6-2. RTP setup for testing.

(A) RTP Global reference frame shown with Y pointing into the page. (B) Close-up of test setup with specimen shown.

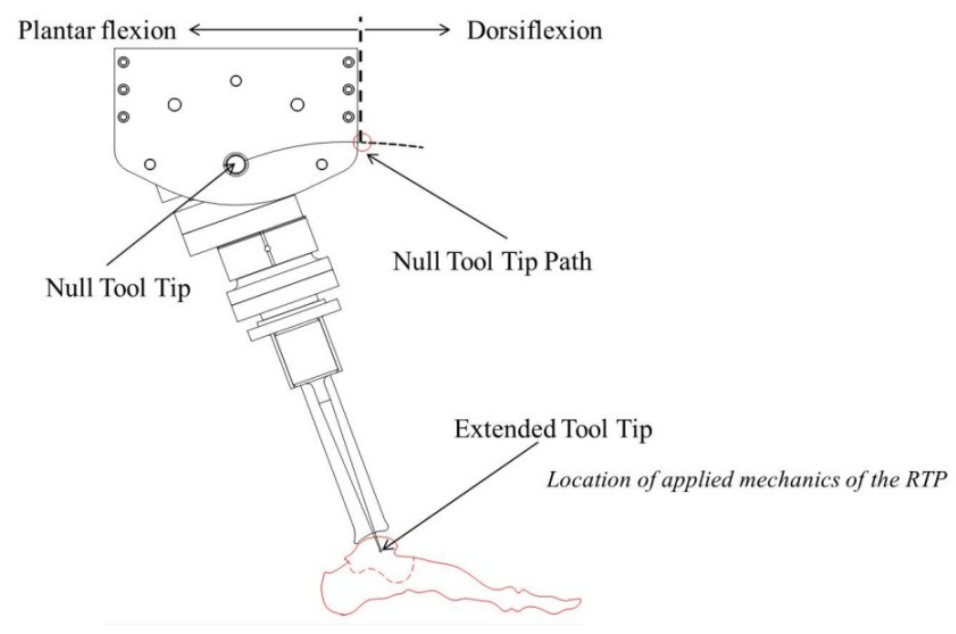

Figure 6-3. Null tool tip and extended tool tip.

This figure identifies the NTT and ETT along with the NTT pathway generated during testing, in this example plantar flexion. 
translation between it and the specimen. The mounting block is then rigidly connected to the RTP.

A cable puller was then attached to the Achilles tendon. The cable puller was placed as low on the tendon as it would go and then a U-bolt was added at the base to increase strength of clamping power. As confirmation of this approach, $1500 \mathrm{~N}$ was applied and held for $60 \mathrm{sec}$ with no slippage witnessed.

Part of the mounting procedure required that the Achilles force be applied with specimen heal not making contact. Use of an X-Y table allows the longitudinal arches to form without resistance. The foot is then lowered until heal makes contact and then required tibia force is applied to meet specific test parameters at the ankle. The X-Y table was then locked for testing.

\section{Testing}

The RTP was used to determine the IAR pathway of the ankle joint from a neutral position of $0^{\circ}$ to $10^{\circ}$ of dorsiflexion and again from a neutral position of 0 to $20^{\circ}$ of plantar flexion with and without a passive Achilles force. The passive force was created by hanging a series of calibrated weights from a cable attached to the Achilles tendon.

A custom software program was written to evaluate a "pure moment" condition when rotating a body about its instantaneous center while maintaining an axial load acting through the tibia. The program functions based on readings of off-axis forces in the $\mathrm{X}$ and $\mathrm{Z}$ axes and precise commanded movements along the same axes.

A torque is applied $\left(\mathrm{M}_{\mathrm{apl}}\right.$, moment applied) about a point to rotate a given amount $(\Delta \theta)$, refer to Figure 6-4. As a result, forces build up in the $X$ and $Z$ axes' about the initial point of rotation, indicating the rotational point is out of position at the end of rotation. The point is then repositioned by taking small incremental steps of $0.25 \mathrm{~mm}$ in the $\mathrm{X}$ and $\mathrm{Z}$ axes to eliminate the off-axis loads, or the effects thereof, so you are left with the actual moment $\left(\mathrm{M}_{\mathrm{act}}\right)$ about the new point or end point. That point is stored and identified as the lowest energy state or point that requires the least amount of torque to rotate $\Delta \theta$. This now reduces the applied moment to a value near the actual moment required to rotate the joint. This process is repeated until the full amount of flexion or extension is achieved. With a resolution of $2.4 \mathrm{~N}$ for $\mathrm{F}_{\mathrm{x}}$ and $\mathrm{F}_{\mathrm{z}}$ and $0.00025 \mathrm{~m}$ for incremental steps taken to relieve off-axis forces, the RTP has a known error of $0.00085 \mathrm{Nm}$ (considered negligible for all practicable purposes) when evaluating the moment about the ankle joint. So, for this study, the RTP was considered as having no impact on the measured results.

\section{Repeatability}

A single test specimen was used to evaluate repeatability of the RTP. Minor precautions were made to eliminate setup errors so the protocol and RTP could be 


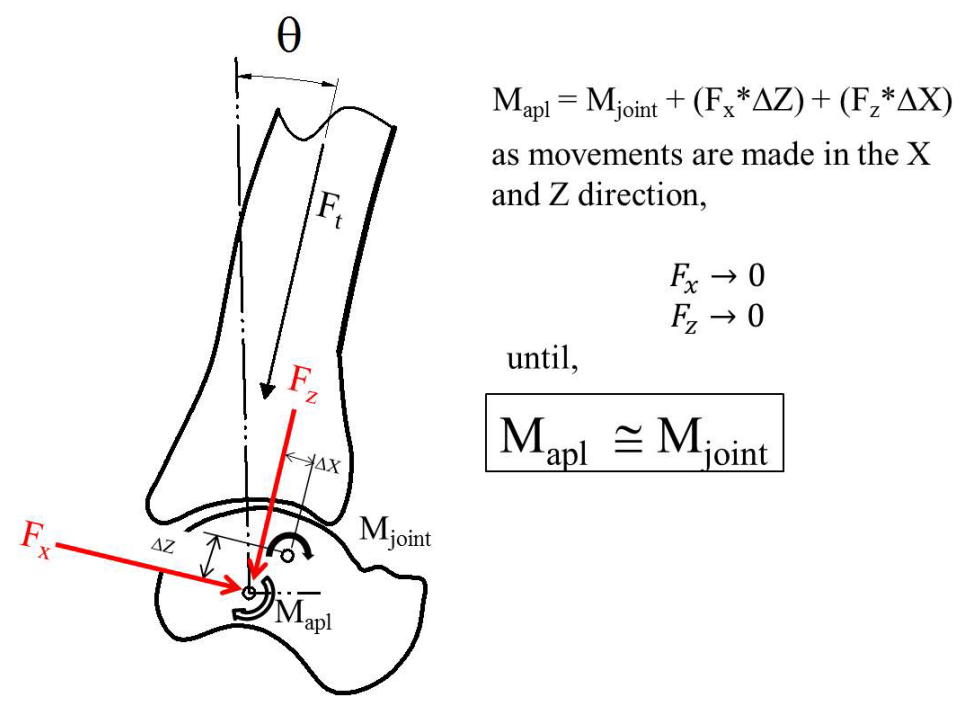

Figure 6-4. Illustration of incremental step in custom testing program.

gauged. Despite this careful approach, relaxation of the tissue was still a factor. The test specimen was cycled five times in each direction, dorsiflexion and plantar flexion. The last three results were used to gauge repeatability.

\section{Results}

The stored data points from the custom program are saved in a text file unique to the test ran. The data contains complete positional data (X, Y, Z, and $\theta$ ) for the NTT and the ETT and forces $\left(F_{x}, F_{y}, F_{z}, M_{x}, M_{y}\right.$, and $\left.M_{z}\right)$ measured about the ETT. These data were used to calculate the IAR of each specimen.

Crisco et al [10] introduced a method for determining the IAR of a rotating mechanism using starting point, ending point and rotational data of one point on a rigid body. Since we have a very accurate measure of the angle rotated and translations in $\mathrm{X}$ and $\mathrm{Z}$ axes per incremental step.

It was necessary to smooth the $\mathrm{X}$ and $\mathrm{Z}$ positional data, each as a function of $\theta$. It was determined that a second order polynomial curve fit was best. The instantaneous axis of rotation (IAR) was then calculated with the smoothed data using Equations 4-1 and 4-2. The IAR pathway was established and is shown in Figure 6-5.

\section{Discussion}

This work addresses the need to evaluate the mechanics, specifically measuring the IAR, of the ankle during normal ROM of during stance phase of gate. Although the 
measurements are in a 2-D sagittal plane and uses a passive Achilles load, this test protocol yields great insight into the mechanics of the ankle joint.

This testing protocol not only confirms previous work $[5,6,7,8]$ that the ankle has an instantaneous axis of rotation, but it is also influenced by Achilles loading. The IAR changes during dorsiflexion and plantar flexion based on an applied Achilles load.

\section{Conclusion}

The RTP yields accurate results of the IAR pathway during average ROM for dorsiflexion and plantar flexion with a passive Achilles load. Also, the RTP is sensitive enough to measure the differences in the IAR pathway varying the Achilles load. Limiting factors for this work are upper load cell capacity, passive loading of Achilles tendon, and locating features for specimen at setup. A higher capacity upper load cell allows us to increase the model to $100 \%$ BW. With means to actively drive the Achilles load (vary with respect to \% of gait cycle) a more accurate understanding of ankle mechanics can be achieved. Better locating features on test fixtures will eliminate setup errors. 

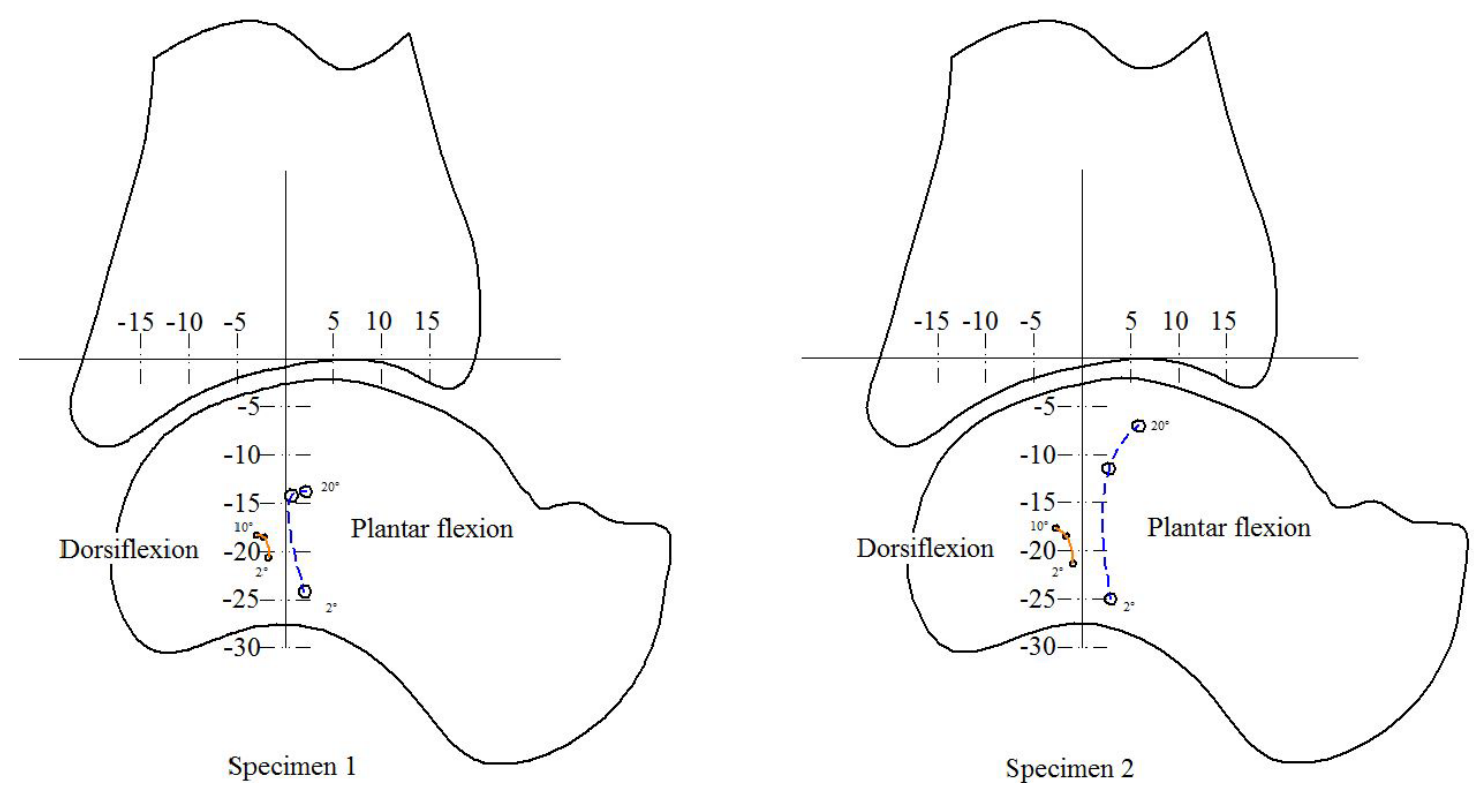

Figure 6-5. Illustration of IAR pathway.

For dorsiflexion, data points of 2, 5, and 10 degrees are plotted and 2, 10, and 20 degrees for plantar flexion. 


\section{CHAPTER 7. DISCUSSION}

Ensuring that the IAR protocol was accurate and repeatable were important steps in the development process of the platform and protocol. Comparing data generated from the protocol against a known data set of a four bar linkage model highlighted the accuracy of this protocol's ability to determine the IAR of a mechanism that behaves similar to the ankle. Over 25 degrees of rotation the maximum true positional error was $0.022 \mathrm{~mm}$ and is considered negligible. However, this error could be reduced by evaluating at smaller angular increments.

Also, a special protocol was established to minimize setup error and tissue relaxation influences during a cycled test. When comparing the last three dorsiflexion curves against each other and the last three plantar flexion curves against each other a repeatable process was revealed. The results showed that the cycled data sets were not significantly different $(\mathrm{P}<0.05)$ for both plantar flexion and dorsiflexion. The platform and protocol proved to be robust and ready for in vitro testing of cadaveric feet.

Plantar flexion seemed relatively uninfluenced by incremental changes in Achilles tendon load. While there are slight shifts of approximately $\pm 1 \mathrm{~mm}$ in the curves, it is unclear as to the exact cause of these shifts. Tissue relaxation, time variations during loading Achilles, and alignment not exact are all setup variables that could cause a slight difference in the results.

Dorsiflexion yielded inconsistent results. The IAR seemed much more unsettled as the Achilles load was incrementally changed, even changing directions between loading conditions. Also, there was a lot of horizontal translation in the IAR pathway at certain loads, which is believed to be relative motion between the talus and tibia. It can be concluded from these results that the talus was constrained, overpowered at certain conditions, and not allowed to move as needed during dorsiflexion.

When no Achilles load is present our findings are similar to the results from Leardini et al [5-8], showing an anterior and slight distal movement of the IAR at passive conditions (no load on the ankle complex). Observed here there is a more pronounced distal slope suggesting relative motion (a translation at the articular surfaces) between the talus and tibia.

However, when the ankle complex is loaded, as noted in Figure 2-3, the IAR pathway for plantar flexion is very different. It has a proximal trend instead of a distal one. Results from Leardini et al [5-8] are based on a passive model (unloaded ankle complex) and assumes the same IAR pathway for loaded and unloaded conditions at the ankle complex. This is based on the assumption that there is little to no change in the lengths of the calcaneofibular and tibiocalcaneal ligaments and that those ligaments serve as a rigid link allowing the ankle complex to be modeled as a 4-bar linkage system. In this study it was concluded that there was a different kinematic profile between loaded and unloaded conditions, which seems to be a factor of activating the medial longitudinal arch. 
Leardini et al [5-8] describe the IAR moving posteriorly and proximally during dorsiflexion. In this study the IAR traveled anteriorly and distally at no load conditions and anteriorly and proximally at 50\% vGRF when the Achilles is loaded. Activating the medial longitudinal arch appears to influence a different kinematic profile in dorsiflexion.

Based on this study, there is a significant difference in the kinematic profile of the ankle complex during plantar flexion and dorsiflexion when the Achilles tendon is unloaded and loaded. The main difference is forming of the longitudinal arch when the Achilles tendon is loaded. Based on the results this has a direct effect on the kinematic profile of both plantar flexion and dorsiflexion. However, once the arch was formed the Achilles tendon load had little to no effect on the kinematic profile of the ankle complex during plantar flexion. In dorsiflexion, the results seemed more unstable, changing shape and direction at different Achilles loads, even with the arch formed. However, in dorsiflexion the kinematic profile of the ankle complex was thought to be stable at $50 \%$ of the vGRF with less relative motion (horizontal translation) between the talus and the tibia.

The results of this study indicates that the ankle rotates about an IAR contained within the sagittal region of the talus and is directly influenced by an Achilles tendon load. The location of the IAR within the talus is a concept strongly supported in the medical community and in studies [4-8] where the instantaneous axis of rotation was of concern. However, the influence that a load on the Achilles tendon has on the IAR is relatively new idea and data on this subject could not be found. 


\section{CHAPTER 8. CONCLUSION}

The RTP was modified to accommodate typical loading conditions the foot and ankle complex will experience during a walking gait. The new gimbal design coupled with the new rotary actuators worked well and is robust enough to apply loads that are seen during the latter part of the stance phase of gait where larger loads act on the ankle joint. The resolution and associated error are extremely low and account for approximately $0.000068 \mathrm{Nm}$ of the applied moment to rotate the tibia about the ankle complex. This accounts for less than $0.01 \%$ of the measured value, so the influence from the RTP on this measurement is considered negligible.

The repeatability results demonstrate a relative accuracy of the RTP and new protocol in determining the IAR of the ankle complex. In dorsiflexion the mean variance was $0.031 \mathrm{~mm}$ and $0.089 \mathrm{~mm}$ in $\mathrm{X}$ and $\mathrm{Z}$ respectively. In plantar flexion the mean variance was $0.044 \mathrm{~mm}$ and $0.300 \mathrm{~mm}$ in $X$ and $Z$ respectively. Also, there is no significance difference $(\mathrm{P}>0.05)$ when comparing the last three cycled runs in dorsiflexion and plantar flexion.

This new testing protocol provides a more accurate approach to determining the IAR of the ankle complex. It is able to analyze the IAR during early stance phase of gait and at a unloaded condition on the Achilles. The results are tight and consistent per results from the repeatability study. While many still estimate the IAR of the ankle complex as a single point [14] or poorly approximated [9-13, 15], this method would offer better insight into the kinetics of the foot and ankle complex. 


\section{CHAPTER 9. LIMITATIONS AND FUTURE WORK}

As with all research there are limitations that need to be addressed to further the science in the field being researched. Here it is the kinetics of the ankle complex with and without an Achilles load present. This research appears to be a new and novel approach to determining the IAR in an in vitro test environment. Thus, eliminating these limitations is critical.

First, the gimbal load cell was limited in capacity. This prevented the ability to apply full BW for the vGRF, instead this work was limited to $50 \% \mathrm{BW}$. Evaluating the ankle complex under full physiological load, $100 \%$ BW, may lead to other results. Either way this needs to be confirmed.

Second, the Achilles load was applied passively. Achilles loading is dynamic in nature. The ability to actively load the Achilles again allows for a more physiological approach for evaluating the ankle complex.

Third, the experimental setup allowed for variation when aligning the specimen. While great care was taken aligning the specimen, this was still a visual process. This could influence the IAR pathway and should be corrected.

Fourth, the data indicated some translation during some of the testing. In the methodology here the ankle complex was fixed while the tibia rotated about it. Dorsiflexion results appeared unstable at certain loading conditions. Understanding the relative motion of the tibia, talus, and calcaneus with respect to each other and the ground would help explain these results.

Last, this work was limited to evaluating the ankle complex during early stance phase of gait. The ankle complex experiences greater dynamic loads at the later part of stance phase of gait. It would best to have a complete data set spanning the full range of stance phase of gait.

Future work to overcome these limitations are configure second robot, establish a physical locating feature on mounting block, increase loading capacity of gimbal load cell (larger load cell), and develop a heel off protocol. Synchronizing robot 1 and robot 2 will allow for actively loading the Achilles and achieve the larger loads experienced during gait. A big part of generating those larger loads will be the heel off protocol. That way the ankle complex will be physiologically positioned as the loads are applied leading to a complete testing methodology for the stance phase of gait. Also, the updated

mounting block will reduce the effects of setup error improving overall repeatability. 


\section{LIST OF REFERENCES}

1. Didomenico, L.A and Camasta, C.A. (2010). Is total ankle replacement more effective than ankle arthrodesis? Podiatry Today. (July)

2. Nordin, M. and V.H. Frankel. (2001). Basic Biomechanics of the Musculoskeletal System. 3rd ed. Philadelphia: Lippincott Williams \& Wilkins. p. 226.

3. Gougoulias, N. E., A. Khanna, et al. (2009). History and evolution in total ankle arthroplasty. Br Med Bull 89: 111-151.

4. Sammarco, G. J., A. H. Burstein, et al. (1973). Biomechanics of the ankle: a kinematic study. Orthop Clin North Am 4(1): 75-96.

5. Leardini, A. (2001). Geometry and mechanics of the human ankle complex and ankle prosthesis design. Clin Biomech 16(8): 706-709.

6. Leardini, A., J. J. O'Connor, et al. (1999). A geometric model of the human ankle joint. J Biomech 32(6): 585-591.

7. Leardini, A., J. J. O'Connor, et al. (1999). Kinematics of the human ankle complex in passive flexion; a single degree of freedom system. J Biomech 32(2): 111-118.

8. Leardini, A., J. J. O'Connor, et al. (2004). Mobility of the human ankle and the design of total ankle replacement. Clin Orthop Relat Res(424): 39-46.

9. Kirane, Y.M., Michelson, J.D, and Sharkey, N.A. (2008). Evidence of isometric function of the flexor hallucis longus muscle in normal gait. J Biomech 41: 19191927.

10. Nester, C.J., Liu, A.M., et al. (2007). In vitro study of foot kinematics using a dynamic walking cadaver model. J Biomech 40(2): 1927-1937.

11. Hurschler, C., Emmerich, J. and Wulker, N. (2003). In vitro simulation of stance phase gait - Part I: Model verification. Foot \& Ankle International, 24(8): 614-622.

12. Kim, K., Kitaoka,H.B., et al. (2001). In Vitro Simulation of the Stance Phase in Human Gait. Journal of Musculoskeletal Research, 5(6): 113-122.

13. Noble, L., Colbrunn, R., et al. (2010). Design and Validation of a General Purpose Robotic Testing System for Musculoskeletal Applications, Journal of Biomechanical Engineering-Transactions of the ASME, 132(2).

14. Baxter,J. R.,T. A. Novack, et al. (2012). Ankle joint mechanics and foot proportions differ between human sprinters and non-sprinters. Proc Biol Sci. 22(1735):2018-24. 
15. Sumiya, T., Y. Suzuki, et al. (1997). Instantaneous centers of rotation in dorsi/plantar flexion movements of posterior-type plastic ankle-foot orthosis. Journal of Rehabilitation Research \& Development 34(3): 279-285.

16. Giddings, V.L., Beaupre, G. S., et al. (2000). Calcaneal loading during walking and running. Medicine \& Science in Sports \& Exercise. 0195-9131/00/3203-0627/0.

17. Kirtley, C. (2005). Clinical gait analysis : theory and practice. Edinburgh ; New York, Elsevier.

18. Winter, D. A. (2009). Biomechanics and Motor Control of Human Movement. 4th ed. John Wiley \& Sons, Inc., Hoboken, NJ.

19. Kelly, B., A. (2005). Multiaxis programmable spine robot for the study of multibody spinal biomechanics using real-time hybrid force and displacement control strategies. Dissertation, The University of Tennessee Health Science Center, Memphis, TN.

20. Crisco JJ I, Chen X, Panjabi MM, et al. (1994). Optimal marker placement for calculating instantaneous center of rotation. J Biomech 27(9):1183-1187.

21. Giannini, S., M. Romagnoli, et al. (2010). Total ankle replacement compatible with ligament function produces mobility, good clinical scores, and low complication rates: an early clinical assessment. Clin Orthop Relat Res 468(10): 2746-2753.

22. Gray, H. and W. H. Lewis. (1918). Anatomy of the human body. Philadelphia, Lea \& Febiger. p.268.

23. Ortho-Worldwide. (2012). Ankle and Foot Bio-Mechanics. http://www.orthoworldwide.com/anfobi.html. Accessed June 1, 2012.

24. Harmonic Drive LLC. (2011). Hollow Shaft Actuators, FHA C series User Manual Catalog. Rev 08. Peabody, MA.

25. Kollmorgen Motions Technologies Group. (2006). Servo Disc Catalog. Commack, NY. 


\section{APPENDIX A. GENERIC GAIT DATA}

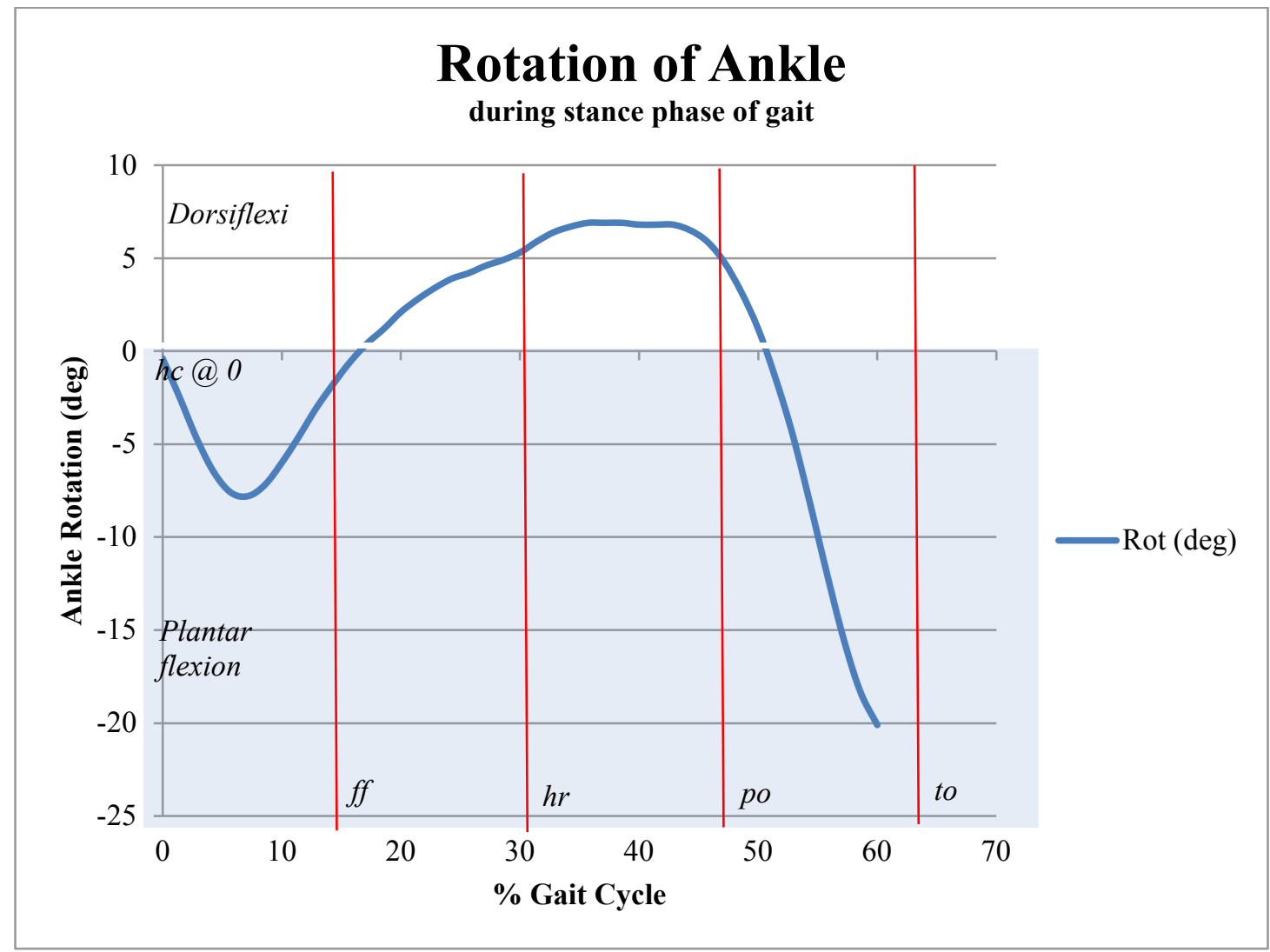

Figure A-1. Graph, ankle rotational data.

This graph represents tabular data for stance phase of gait. Source: Winter, D. A. (2009). Biomechanics and motor control of human movement. Hoboken, N.J., Wiley. 


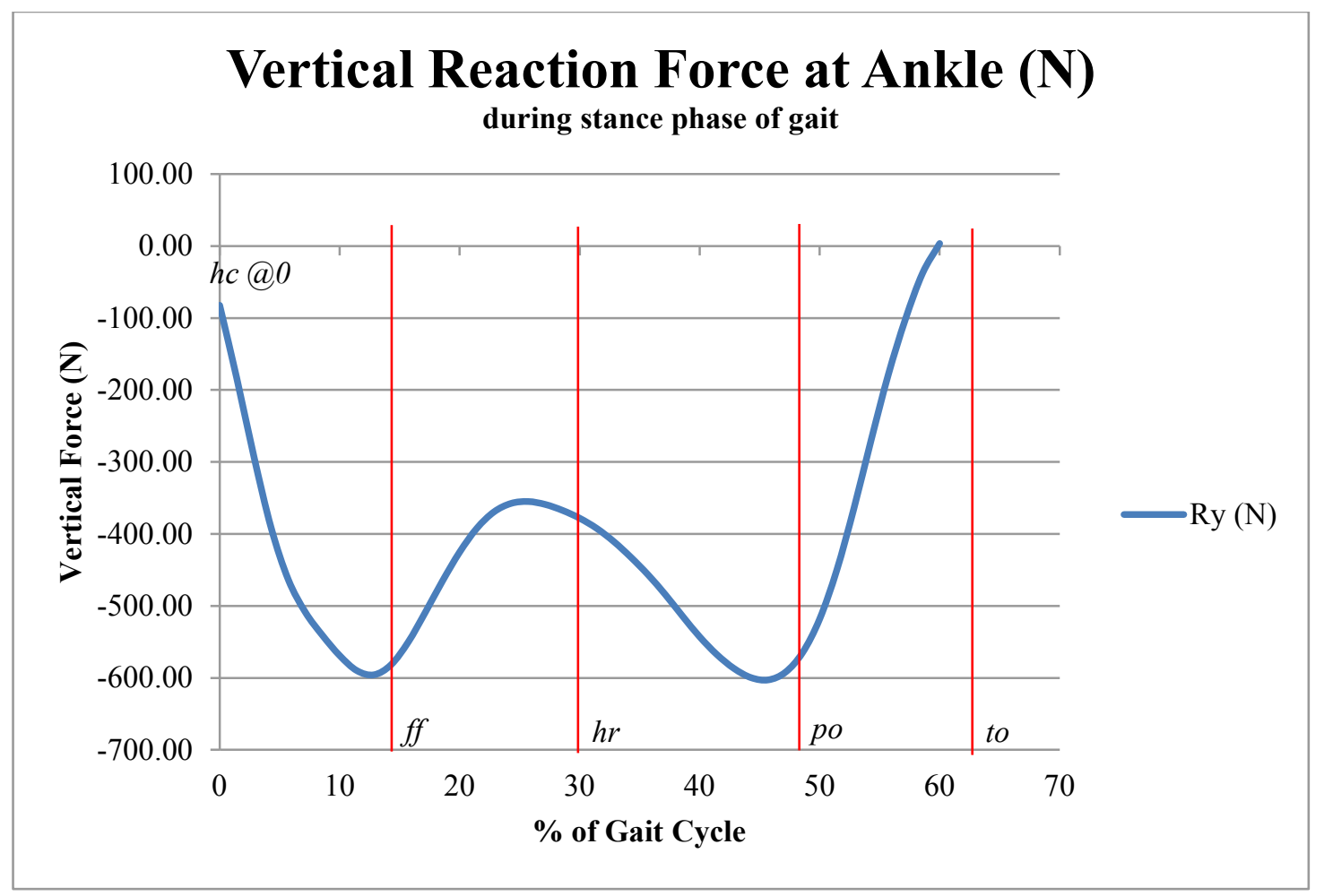

Figure A-2. Graph, vGRF data.

This graph represents tabular data for stance phase of gait. Source: Winter, D. A. (2009). Biomechanics and motor control of human movement. Hoboken, N.J., Wiley. 


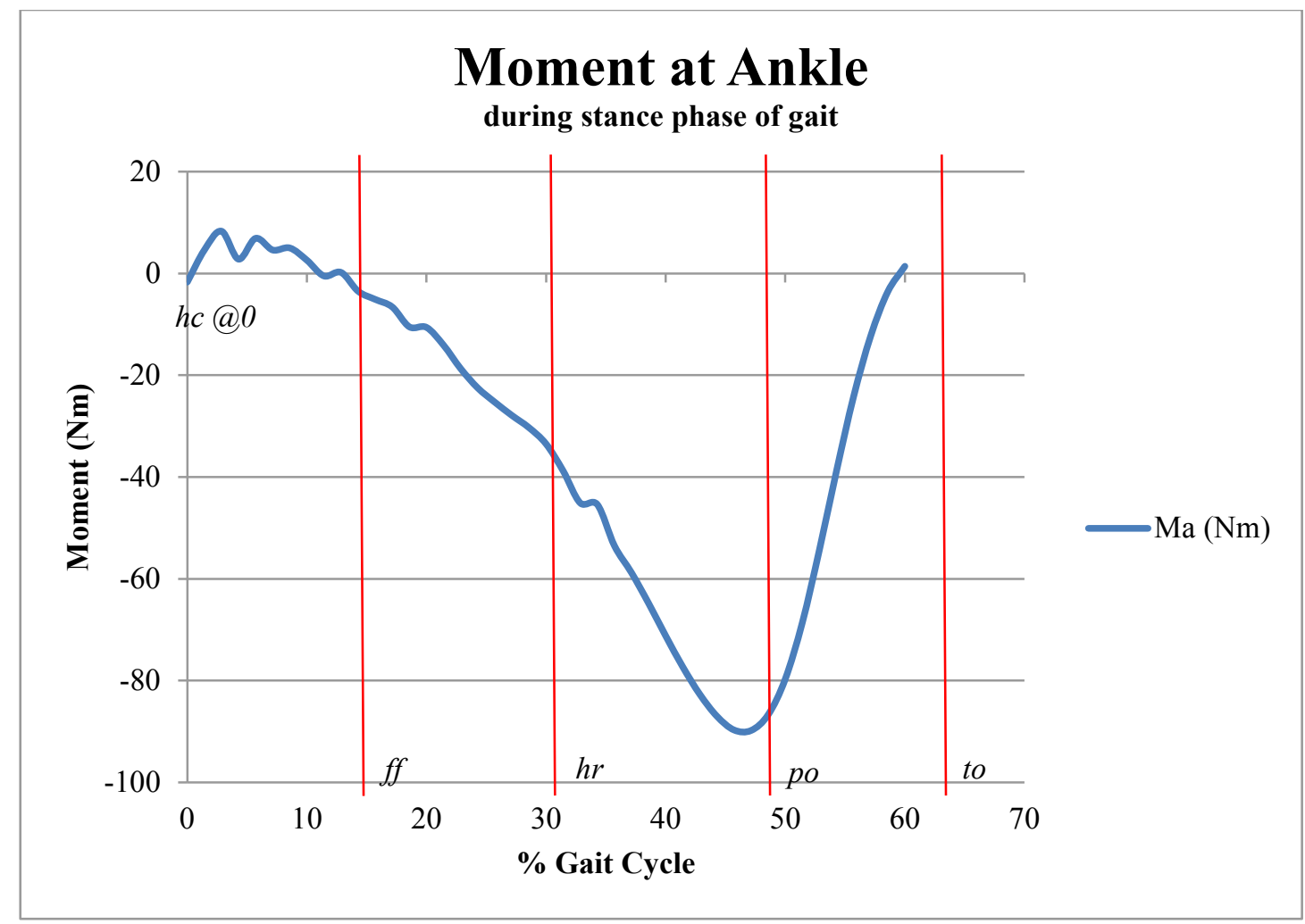

Figure A-3. Graph, moment at ankle.

This graph represents tabular data for stance phase of gait. Source: Winter, D. A. (2009). Biomechanics and motor control of human movement. Hoboken, N.J., Wiley. 


\section{APPENDIX B. TRANSFORMATIONS, TOOL TIP AND FORCE FRAME}

Dimensions 175.4 \& 77.2 are hard-coded into the program so that all transformations are made from a common reference plane

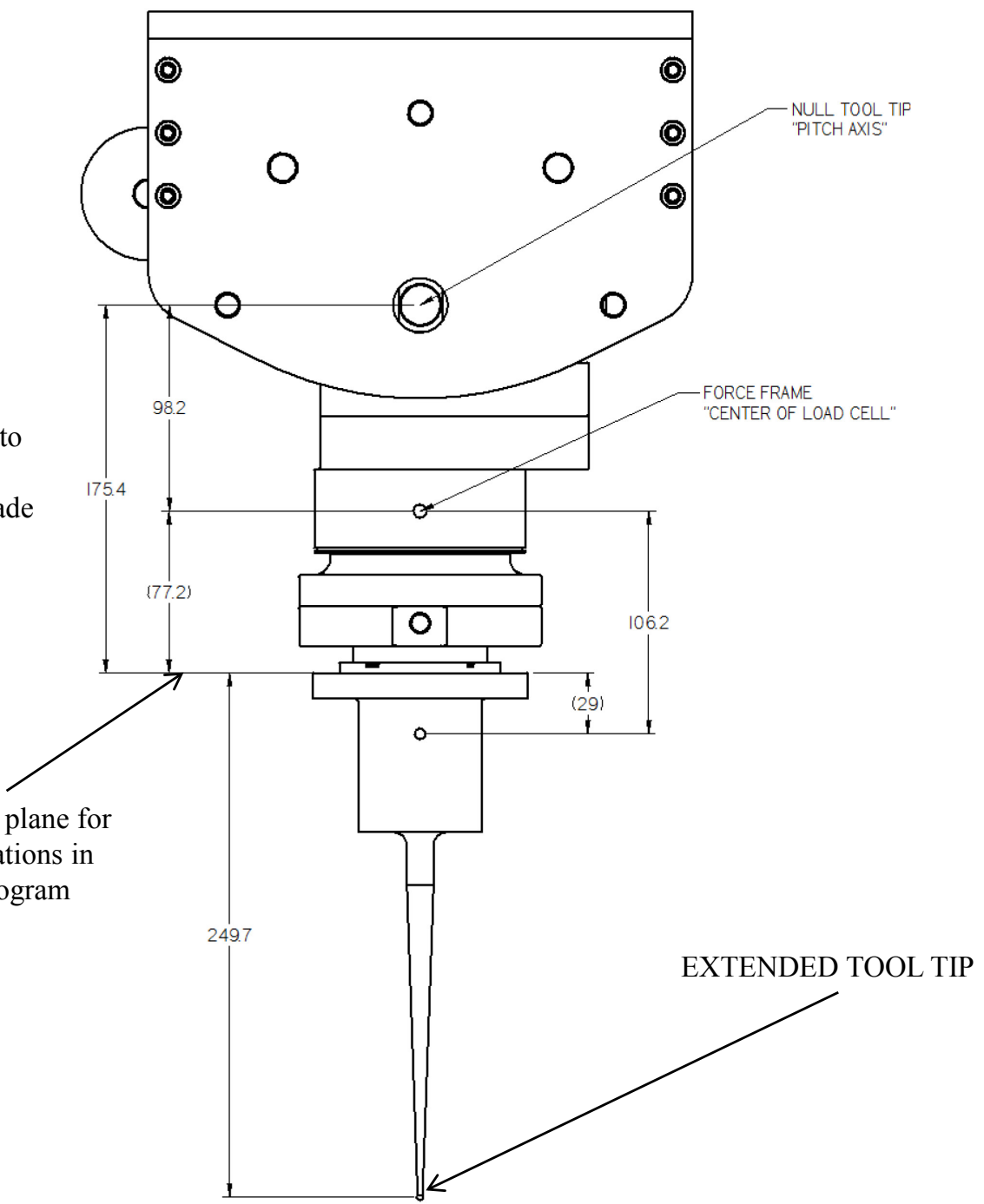

Figure B-1. Tool tip transformations, dimensions in $\mathbf{m m}$.

As illustrated here, 249.7 would be entered into the program for transforming the null tool tip straight down and establishing the extended tool tip. The same 249.7 is used to transform the force frame to the extended tool tip to measure forces at that point. 


\section{APPENDIX C. DATA PROCESSING, POLYNOMIAL FIT, SMOOTHING, AND IAR CALCULATIONS}

First, null tool tip data from the RTP during testing is imported into Mathcad. Included data are rotation about pitch axis $(\theta) \underline{\text { column } 0}$, translation along $\mathrm{X}$-axis $(\mathrm{X})$ column 1 , and translation along Z-axis $(Z)$ column 2 . Polynomial regression is performed on $\mathrm{X}$ and $\mathrm{Z}$ data as a function of $\theta$ to establish a smooth data set for $\mathrm{X}$ and $\mathrm{Z}$ to minimize erratic results in IAR calculations.

data $=$
\begin{tabular}{rr|r|r|r|}
\hline & 0 & 1 & 2 \\
\hline 0 & 0.1008 & 0 & 0 \\
\hline 1 & 0.5002 & -2.612 & -0.0137 \\
\hline 2 & 0.9999 & -5.884 & -0.0564 \\
\hline 3 & 1.5001 & -9.158 & -0.1277 \\
\hline 4 & 2 & -12.4301 & -0.2276 \\
\hline 5 & 2.5 & -15.702 & -0.3562 \\
\hline 6 & 2.9999 & -18.972 & -0.5134 \\
\hline 7 & 3.5001 & -22.2379 & -0.6412 \\
\hline 8 & 4 & -25.504 & -0.8554 \\
\hline 9 & 4.5 & -28.77 & -1.0979 \\
\hline 10 & 4.9999 & -32.0319 & -1.3689 \\
\hline 11 & 5.4999 & -35.292 & -1.6684 \\
\hline 12 & 6.0001 & -38.5499 & -1.9964 \\
\hline 13 & 6.5 & -41.8021 & -2.3527 \\
\hline 14 & 7 & -45.054 & -2.7375 \\
\hline 15 & 7.4999 & -48.302 & $\ldots$ \\
\hline
\end{tabular}

$\begin{array}{lll}\theta:=\text { data }^{\langle 0\rangle} & \text { establishes data set for } \theta & \text { range variables } \\ \mathrm{X}:=\text { data }^{\langle 1\rangle} & \text { establishes data set for } \mathrm{X} & \mathrm{i}:=0 . .9 \\ \mathrm{Z}:=\text { data }^{\langle 2\rangle} & \text { establishes data set for } \mathrm{Z} & \mathrm{j}:=0,1 . .39 \\ & \mathrm{t}:=0 . .20\end{array}$

determines incremental rotation in degrees

$\phi_{\mathrm{j}}:=\left(\theta_{\mathrm{j}+1} \cdot \operatorname{deg}-\theta_{\mathrm{j}} \cdot \mathrm{deg}\right)$ 
This section evaluates variance of data set vs order of polynomial; the following "polyerx" program is from the data analysis extension pack of Mathcad (2007).

$$
\text { polyerx }:=\mid \begin{aligned}
& \text { for } n \in 1 . .10 \\
& \mid \begin{array}{l}
\mathrm{c} \leftarrow \operatorname{regress}(\theta, X, n) \\
\operatorname{SSE}_{\mathrm{n}-1} \leftarrow \frac{1}{\operatorname{length}(\theta)-\mathrm{n}} \cdot \sum_{\mathrm{j}=0}^{\operatorname{last}(\theta)}\left(X_{j}-\operatorname{interp}\left(\mathrm{c}, \theta, \mathrm{X}, \theta_{\mathrm{j}}\right)\right)^{2}
\end{array}
\end{aligned}
$$

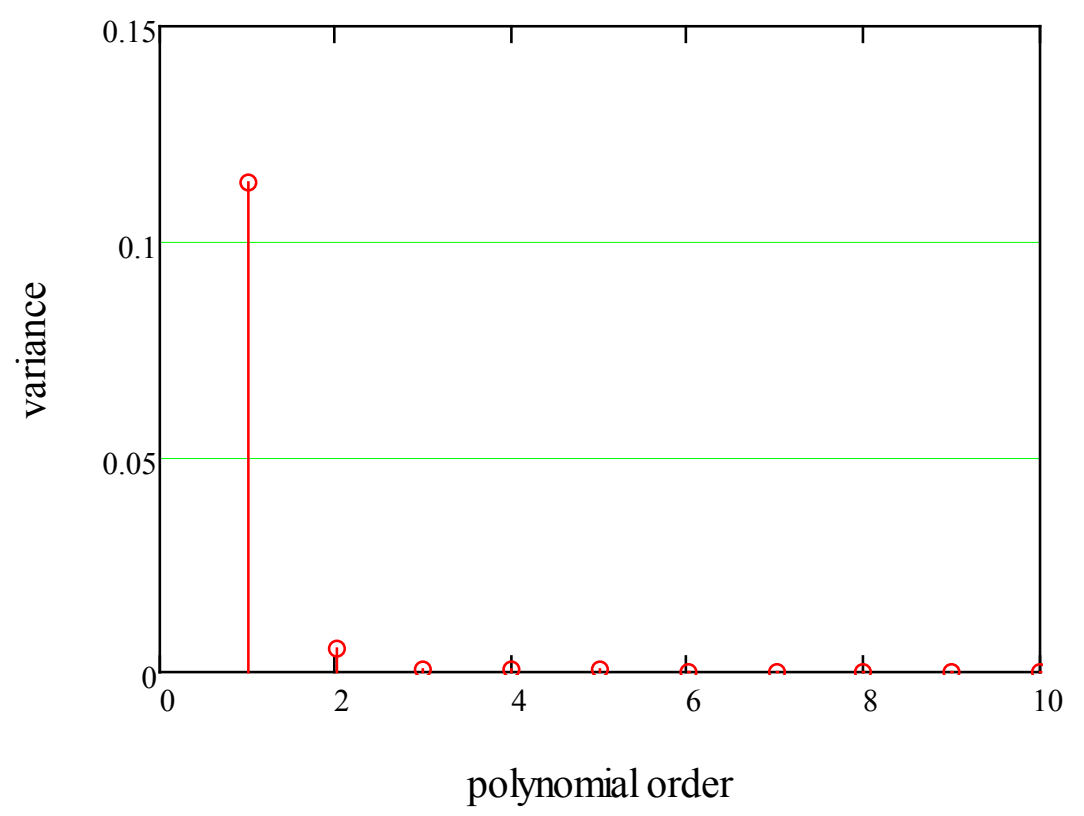

$\begin{aligned} & \text { polyerx }^{\mathrm{T}}= \begin{array}{|c|c|c|c|c|c|c|}\hline & 0 & 1 & 2 & 3 & 4 & 5 \\ \hline 0 & 0.1138 & 5.5784 \cdot 10-3 & 5.7603 \cdot 10-4 & 4.4505 \cdot 10-4 & 3.4607 \cdot 10-4 & \ldots \\ \hline\end{array} \\ & \operatorname{polyerx}_{2}=0.000576\end{aligned}$

There is no significant gain in accuracy in a polynomial greater than a second order polynomial. 
This section establishes a second order polynomial to represent $X(\theta)$. This also smoothes the data and improves the quality of IAR calculations.

The regress function determines polynomial coefficients, that minimize the sum of squares equation

$$
\sum_{j=0}^{N}\left[Y_{j}-\sum_{i=0}^{n}\left[c_{i} \cdot\left(X_{j}\right)^{i}\right]\right]^{2}
$$

by differentiating with respect to each coefficient and setting results equal to zero. The interp function returns a single interpolated $\mathrm{X}$ value for a given $\theta$ value.

$$
\mathrm{c} 2 \mathrm{x}:=\operatorname{regress}(\theta, \mathrm{X}, 2) \quad \mathrm{p} 2 \mathrm{x}(\mathrm{t}):=\operatorname{interp}(\mathrm{c} 2 \mathrm{x}, \theta, \mathrm{X}, \mathrm{t})
$$

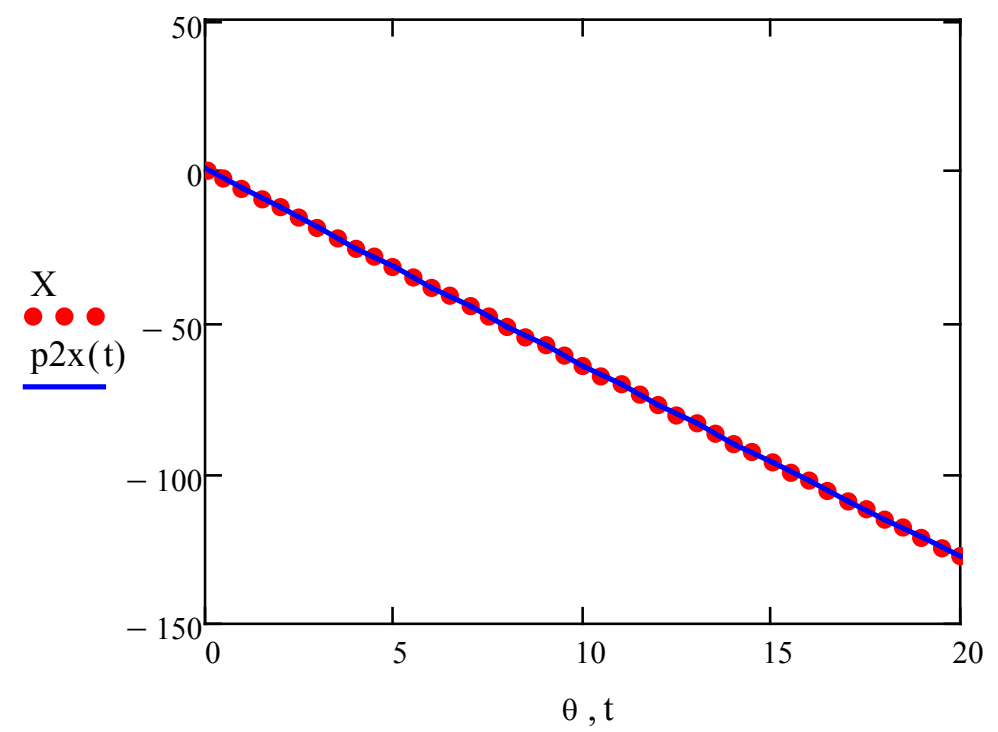

The red dots represents the actual data points and the solid blue line the best fit function.

$\mathrm{c} 2 \mathrm{x}^{\mathrm{T}}=\left(\begin{array}{llllll}3 & 3 & 2 & 0.8238 & -6.6416 & 0.0104\end{array}\right)$

The last three terms are the coefficients of the fitting polynomial from lowest to highest degree terms.

2nd order term

$$
\begin{aligned}
& \mathrm{a}:=\mathrm{c} 2 \mathrm{x}_{5} \\
& \mathrm{a}=0.0104
\end{aligned}
$$

1 st order term

$\mathrm{b}:=\mathrm{c} 2 \mathrm{x}_{4}$

$\mathrm{b}=-6.6416$
0 order term

$\mathrm{d}:=\mathrm{c} 2 \mathrm{x}_{3}$

$\mathrm{d}=0.8238$ 
The resulting function for $\mathrm{X}(\theta)$ is:

$X(\theta)=a \cdot \theta^{2}+b \cdot \theta+d$

Repeat for $Z(\theta)$ :

evaluate variance vs order of polynomial

polyerz $:=\mid \begin{aligned} & \text { for } \mathrm{n} \in 1 . .10 \\ & \begin{array}{l}\mathrm{c} \leftarrow \operatorname{regress}(\theta, \mathrm{Z}, \mathrm{n}) \\ \mathrm{SSE}_{\mathrm{n}-1} \leftarrow \frac{1}{\operatorname{length}(\theta)-\mathrm{n}} \cdot \sum_{\mathrm{j}=0}^{\operatorname{last}(\theta)}\left(\mathrm{Z}_{\mathrm{j}}-\operatorname{interp}\left(\mathrm{c}, \theta, \mathrm{Z}, \theta_{\mathrm{j}}\right)\right)^{2}\end{array}\end{aligned}$

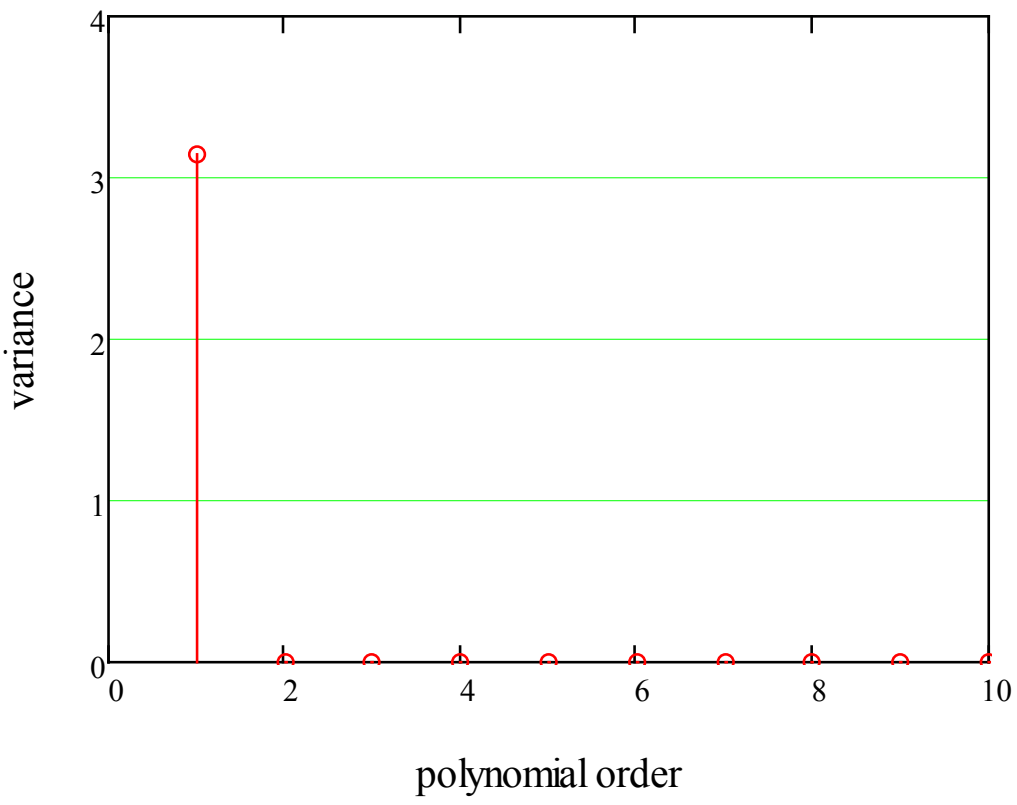

polyerz $^{\mathrm{T}}=$\begin{tabular}{|c|c|c|c|c|c|}
\hline & 0 & 1 & 2 & 3 & 4 \\
\hline 0 & 3.1398 & $9.0722 \cdot 10^{-4}$ & $8.4608 \cdot 10^{-4}$ & $8.6574 \cdot 10^{-4}$ & $\ldots$ \\
\hline
\end{tabular}

polyerz $_{2}=0.000846$ 
There is no significant gain in accuracy in a polynomial greater than a second order polynomial.

$$
\mathrm{c} 2 \mathrm{z}:=\operatorname{regress}(\theta, \mathrm{Z}, 2) \quad \mathrm{p} 2 \mathrm{z}(\mathrm{t}):=\operatorname{interp}(\mathrm{c} 2 \mathrm{z}, \theta, \mathrm{Z}, \mathrm{t})
$$

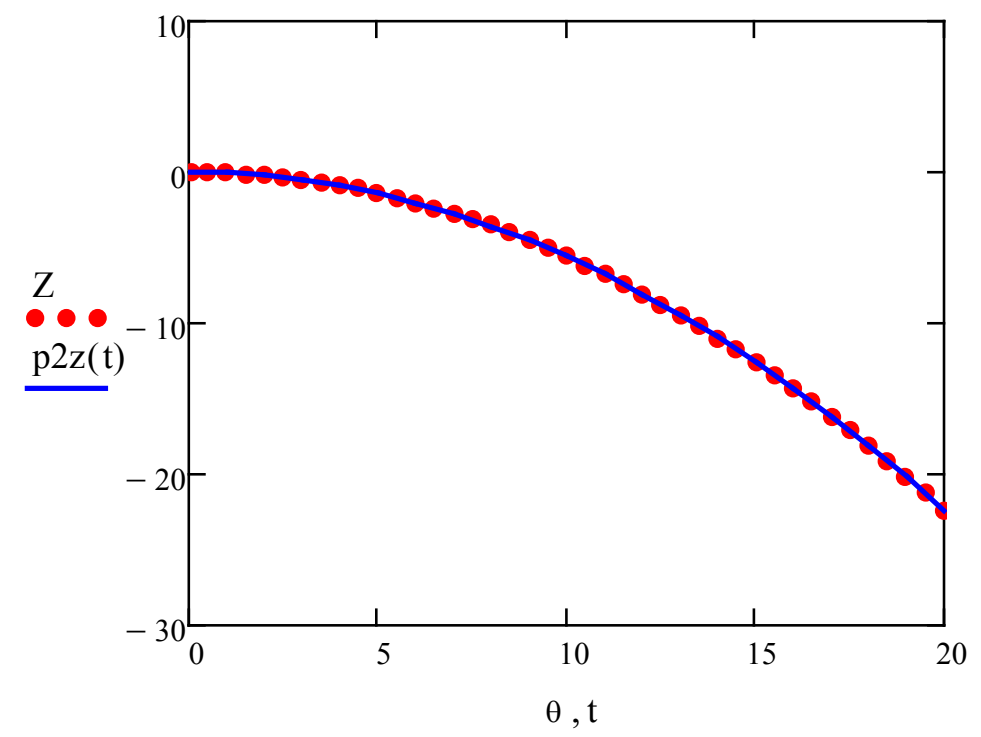

The red dots represents the actual data points and the solid blue line the best fit function.

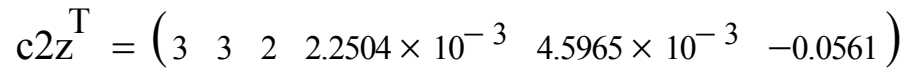

2nd order term

$\mathrm{f}:=\mathrm{c} 2 \mathrm{z}_{5}$

$\mathrm{f}=-0.0561$
1 st order term

$\mathrm{h}:=\mathrm{c} 2 \mathrm{z}_{4}$

$\mathrm{h}=4.5965 \times 10^{-3}$
0 order term

$\mathrm{k}:=\mathrm{c} 2 \mathrm{z}_{3}$

$\mathrm{k}=2.2504 \times 10^{-3}$

$X s:=a \cdot \theta^{2}+b \cdot \theta+d$

$\mathrm{Xs}^{\mathrm{T}}=$\begin{tabular}{|l|l|l|l|l|l|l|}
\hline & 0 & 1 & 2 & 3 & 4 & 5 \\
\hline 0 & 0.1544 & -2.4958 & -5.8068 & -9.1159 & -12.4178 & $\ldots$ \\
\hline
\end{tabular}

$\mathrm{Zs}:=\mathrm{f} \cdot \theta^{2}+\mathrm{h} \cdot \theta+\mathrm{k}$

$\mathrm{Zs}^{\mathrm{T}}=$\begin{tabular}{|c|c|c|c|c|c|}
\hline & 0 & 1 & 2 & 3 & 4 \\
\hline 0 & $2.1434 \cdot 10^{-3}$ & $-9.4944 \cdot 10^{-3}$ & -0.0493 & -0.1172 & $\ldots$ \\
\hline
\end{tabular}


Given the positional data of the null tool tip, the IAR of the ankle will be calculated. Crisco et al (1994) devised a method to determine the IAR of a mechanism tracking a single point, given rotation, and beginning and ending coordinates of the point are known.

Crisco et al equations:

$$
\begin{aligned}
& X_{\mathrm{iar}}=\frac{1}{2} \cdot\left(\mathrm{X}_{1}+\mathrm{X}_{2}\right)+\frac{\left(\mathrm{Z}_{1}-\mathrm{Z}_{2}\right) \cdot \sin (\phi)}{2 \cdot(1-\cos (\phi))} \\
& Z_{\text {iar }}=\frac{1}{2} \cdot\left(Z_{1}+Z_{2}\right)-\frac{\left(X_{1}-X_{2}\right) \cdot \sin (\phi)}{2 \cdot(1-\cos (\phi))} \\
& X_{\text {iar }_{j}}:=\frac{1}{2} \cdot\left(X_{s_{j}}+X s_{j+1}\right)+\frac{\left(Z s_{j}-Z s_{j}+1\right) \cdot \sin \left(\phi_{j}\right)}{2 \cdot\left(1-\cos \left(\phi_{j}\right)\right)} \\
& Z_{\text {iar }_{j}}:=\frac{1}{2} \cdot\left(Z s_{j}+Z s_{j+1}\right)-\frac{\left(X s_{j}-X s_{j+1}\right) \cdot \sin \left(\phi_{j}\right)}{2 \cdot\left(1-\cos \left(\phi_{j}\right)\right)}
\end{aligned}
$$




\section{VITA}

Thomas Edgar Stewart was born in Jesup, Georgia in 1968. He was raised in Jesup as well and graduated from Wayne County High School in 1986. After graduation he enlisted in the US Navy and was honorably discharged four years later. He attended Mississippi State University and graduated cum laude with a Bachelors of Science in Mechanical Engineering December 1997. After many years working in industry he returned to school and earned a Master of Science in Biomedical Engineering, focusing on biomechanics of the foot and ankle complex, from the University of Tennessee Health Science Center. 